\title{
Resonant Excitation and Purcell Enhancement of Coherent Nitrogen-Vacancy Centers Coupled to a Fabry-Perot Microcavity
}

\author{
M. Ruf $\odot,{ }^{1,2, \dagger}$ M.J. Weaver $\oplus^{1,2, \dagger}$ S.B. van Dam $\oplus^{1,2}$ and R. Hanson $\circledast^{1,2, *}$ \\ ${ }^{1}$ QuTech, Delft University of Technology, 2628 CJ Delft, The Netherlands \\ ${ }^{2}$ Kavli Institute of Nanoscience, Delft University of Technology, 2628 CJ Delft, The Netherlands
}

(Received 11 September 2020; revised 14 December 2020; accepted 17 December 2020; published 19 February 2021)

\begin{abstract}
The nitrogen-vacancy $(\mathrm{N}-V)$ center in diamond has been established as a prime building block for quantum networks. However, scaling beyond a few network nodes is currently limited by low spin-photon entanglement rates, resulting from the $\mathrm{N}-V$ center's low probability of coherent photon emission and collection. Integration into a cavity can boost both values via the Purcell effect, but poor optical coherence of near-surface N- $V$ centers has so far prevented their resonant optical control, as would be required for entanglement generation. Here, we overcome this challenge, and demonstrate resonant addressing of individual, fiber-cavity-coupled N- $V$ centers, and collection of their Purcell-enhanced coherent photon emission. Utilizing off-resonant and resonant addressing protocols, we extract an enhancement of the zero-phonon line emission by a factor of up to 4, consistent with a detailed theoretical model. This model predicts that the probability of coherent photon detection per optical excitation can be increased to $10 \%$ for realistic parameters - an improvement over state-of-the art solid immersion lens collection systems by 2 orders of magnitude. The resonant operation of an improved optical interface for single coherent quantum emitters in a closed-cycle cryogenic system at $T \sim 4 \mathrm{~K}$ is an important result towards extensive quantum networks with long coherence.
\end{abstract}

DOI: 10.1103/PhysRevApplied.15.024049

\section{INTRODUCTION}

Future large-scale quantum networks sharing entanglement between their nodes may enable a suite of applications, such as secure communication, distributed quantum computation, and quantum enhanced sensing [1-5]. These networks require nodes with both access to long-lived memory qubit registers that can be operated with high fidelity, and bright spin selective optical transitions with good coherence [2]. Promising node candidates include group IV defects in diamond [6-8], defects in $\mathrm{SiC}$ [9-11], rare earth ions in solid-state hosts [12-14], quantum dots $[15,16]$, neutral atoms $[17,18]$, and trapped ions $[19,20]$.

The nitrogen-vacancy $(\mathrm{N}-V)$ center in diamond combines optical transitions suitable for remote entanglement generation under moderate cryogenic conditions with outstanding electron spin coherence $\left(T_{2}>1 \mathrm{~s}\right)$ and extensive

\footnotetext{
${ }^{*}$ Correspondence and requests for materials should be addressed to m.t.ruf@tudelft.nl or r.hanson@tudelft.nl

${ }^{\dagger}$ These authors contributed equally to this work.

Published by the American Physical Society under the terms of the Creative Commons Attribution 4.0 International license. Further distribution of this work must maintain attribution to the author(s) and the published article's title, journal citation, and DOI.
}

control capabilities over local ${ }^{13} \mathrm{C}$ memory atoms $[21,22]$; these features have enabled pioneering quantum network experiments [23,24] and fundamental tests of physics [25]. However, entanglement generation rates are limited by the relatively low photon emission into the zero-phonon line (ZPL), as well as low collection efficiency from diamond, hindering scaling beyond a few nodes. Both values can be significantly increased by embedding the $\mathrm{N}-V$ center inside an optical cavity, making use of the Purcell effect. Cavity-coupling of $\mathrm{N}-V$ centers at low temperature has been demonstrated for different cavity implementations, including photonic crystals [26-31], microdisk resonators [32], and open microcavities [33,34].

Entanglement generation between nodes requires coherent photon detection after resonant excitation of the optical transitions of individual $\mathrm{N}-V$ centers [21]. However, poor optical coherence (approximately gigahertz linewidths), resulting from surface noise effects and/or implantationinduced damage, has so far prevented resonant optical addressing of Purcell-enhanced N- $V$ centers $[26,29,31,33$, $35,36]$. This has presented a critical roadblock on the path towards remote entanglement generation.

In this work, we demonstrate resonant optical addressing of Purcell-enhanced N- $V$ centers coupled to a fiberbased cavity, capitalizing on recent breakthroughs in diamond membrane fabrication [37] and diamond-based open 
microcavities [33,38-43]. We outline the experimental system in Sec. II, and begin experiments by verifying Purcell enhancement under off-resonant excitation in Sec. III. Next, we develop and demonstrate a resonant excitation protocol for $\mathrm{N}-V$ centers in the cavity. We use the resonant addressing to measure optical coherence (Sec. IV) and Purcell enhancement (Sec. V). Finally, in Sec. VI, we quantify the Purcell-enhanced photon emission and the collection efficiency from a single quantum emitter. We conclude with an outlook including future prospects and avenues opened up by this work.

\section{EXPERIMENTAL SETUP}

An overview of the experimental setup used in this work can be seen in Fig. 1(a). At the heart of the experiment is an open, fiber-based Fabry-Perot microcavity (design finesse 6200), formed from a flat, super-polished mirror, and a laser ablated fiber mirror [44]; this finesse value is chosen as it maximizes the outcoupled fraction of photons emitted into the ZPL for the vibrations present in the cavity [45] [approximately a $(0.1-0.2) \mathrm{nm}$ root-mean-squared amplitude; for a detailed discussion, see Appendix E 1]. The fiber sits on top of a piezo positioning stage, which enables in situ tuning of the cavity position and length under operation in a closed-cycle cryostat $(T \sim 4 \mathrm{~K}$ for all measurements in this paper). Excitation light is delivered to the cavity via the fiber mirror, while all detection in this work takes place in free space through the flat mirror of the cavity. Unbalanced mirror coatings set the design finesse almost entirely by transmission through this flat cavity mirror; for a full overview of the experimental setup, see Appendix A. An electron irradiated and annealed diamond membrane is bonded to the flat cavity mirror [40], and etched down to a final thickness of approximately $5.8 \mu \mathrm{m}$ in the cavity region, following the process flow developed in Ref. [37]. Importantly, this recipe has been shown to preserve the optical coherence of N- $V$ centers needed for entanglement generation, even for a few-micrometer thin diamond samples.

We start characterizing the coupled system of the membrane and fiber cavity by recording cavity spectra under illumination with a broadband light source for different cavity lengths. From fits to a transfer matrix model [38], we infer a membrane thickness of about $5.8 \mu \mathrm{m}$ and an air gap of about $7 \mu \mathrm{m}$ (see Appendix B). This air gap could not be reduced further, likely due to a piece of dust on, or an angled mounting of, the fiber. This limits the cavity finesse to about $2000\left(Q \sim 1.3 \times 10^{5}\right)$ due to operation in the clipping loss regime of the fiber mirror [44,45]. The cavity quantum electrodynamics parameters $(\mathrm{N}-V$ center optical transition linewidth [37], N- $V$-cavity coupling rate, and cavity decay rate $\{\gamma, g, \kappa\} \sim 2 \pi \times\{18 \mathrm{MHz}, 35 \mathrm{MHz}$, $3.5 \mathrm{GHz}\})$ place the system in the weak coupling regime, which is favorable for photon collection (Appendix B).

We find $\mathrm{N}-V$ centers by scanning a fundamental cavity mode over the ZPL transition frequencies (about $470.4 \mathrm{THz}$ ), while constantly illuminating with offresonant $(515 \mathrm{~nm})$ laser light. At this laser wavelength, the optical excitation keeps the $\mathrm{N}-V$ center predominantly in the negative charge and $m_{s}=0$ spin state. In Fig. 1(b) we show the fluorescence counts in a window of $\pm 2 \mathrm{~nm}$
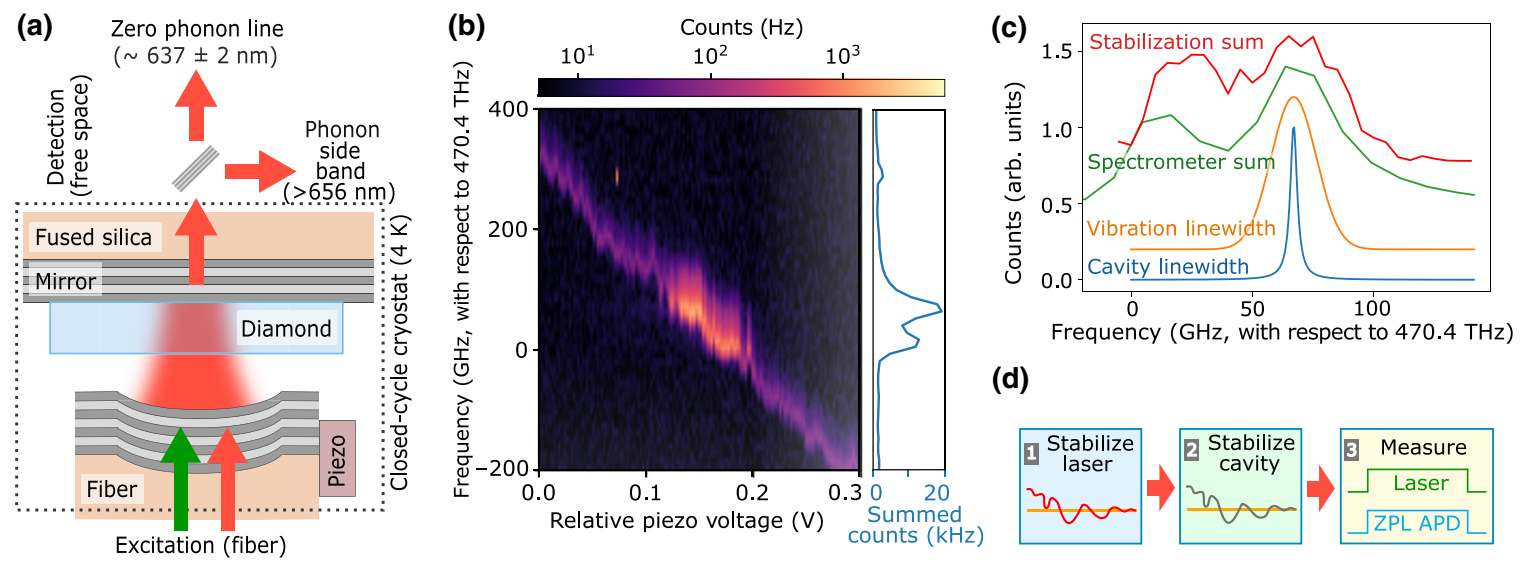

(d)

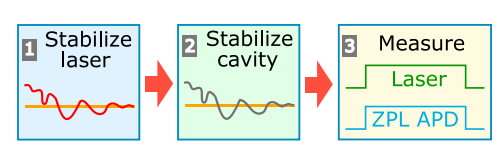

FIG. 1. Experimental setup, N- $V$-cavity characterization measurements, and measurement sequence. (a) Overview of the experimental setup. A tunable fiber-based microcavity with embedded diamond membrane is formed inside a closed-cycle cryostat operated at a temperature of $4 \mathrm{~K}$. (b) Fluorescence counts for different applied piezo voltages (and thus cavity lengths) under off-resonant excitation, detected with a spectrometer in a $\pm 2 \mathrm{~nm}$ window around the $\mathrm{N}-V$ center ZPL of $637 \mathrm{~nm}$ (integration time $5 \mathrm{~s}$ per point). Right panel shows data on the left, summed over all piezo voltages. (c) From bottom to top, measurements of intrinsic cavity linewidth, inferred vibrations-broadened cavity linewidth, spectrometer peaks [same data as the right panel of (b)], and stabilization curve, using the continuous off-resonant excitation and ZPL detection measurement sequence displayed in (d). (d) General measurement sequence used throughout this paper. An $637 \mathrm{~nm}$ laser is frequency stabilized to a wavemeter (1), and serves as a reference for the cavity length (2). Measurement blocks that are repeated multiple times are interleaved between stabilization rounds (3). 
around the expected ZPL frequency on a spectrometer for different piezo voltages (and thus cavity lengths). Background light in the cavity serves as an internal light source [46], revealing the expected decrease in cavity frequency with increasing voltage. As the cavity mode is tuned through the ZPL transition frequencies of a small ensemble of $\mathrm{N}-V$ centers, additional $\mathrm{N}-V$ center fluorescence is collected. Summing the spectrometer fluorescence counts per frequency over all cavity lengths reveals two peaks [right panel of Fig. 1(b)], which are likely due to the $E_{x}$ and $E_{y}$ transitions (see Sec. IV). Each peak is significantly wider than the intrinsic cavity linewidth.

To investigate the origin of the observed width of the $\mathrm{N}-V$ center emission peaks, we compare the intrinsic cavity linewidth [blue trace in Fig. 1(c)] - measured on a timescale much faster than the vibrations in the cavity (Appendix B) - to the "vibration linewidth," calculated by convolving the measured vibration value with the intrinsic cavity linewidth [orange trace in Fig. 1(c)]. The resulting curve, which is an estimate for the linewidth averaged over during the full spectrometer run, explains most of the width of the peaks in the summed spectrometer data [green trace in Fig. 1(c)]; the additional width is attributed to a frequency distribution of $\mathrm{N}-V$ centers in the cavity (see Sec. IV).

For more refined cavity control, we design a measurement protocol that we use throughout the remainder of this paper [see Fig. 1(d)]. The sequence starts by stabilizing an $637 \mathrm{~nm}$ laser to a given setpoint using a wavemeter. We then stabilize the cavity to this laser frequency, after which we start a measurement sequence. By repeating this procedure, we can take out slow drifts between measurements. We test this measurement protocol by stabilizing the cavity to different laser setpoints and taking fluorescence data in the ZPL under off-resonant excitation [red trace in Fig. 1(c)]. The resulting data are consistent with the lineshape found via the spectrometer measurement.

\section{OFF-RESONANT EXCITATION}

Entanglement generation rates in quantum networks scale with the collection of coherent photon emission. A cavity acts as a spectral filter, only allowing resonant emission to exit, and opens up an additional decay channel for the excited state of any coupled $\mathrm{N}-V$ centers, which in turn decreases their lifetime. Thus, when the cavity is resonant with a $\mathrm{N}-V$ center optical transition, more ZPL light should be emitted with a reduced lifetime, as has been observed in different cryogenic systems [26-33]. Importantly, this additional decay is also funneled into the cavity mode that can be readily collected, as opposed to free-space systems, in which the ZPL light is emitted in all directions. From the reduced lifetime, one can (following the definition in Refs. [26-29]) extract the Purcell enhancement induced by

the cavity, $F_{P}^{\mathrm{ZPL}}$, as

$$
F_{P}^{\mathrm{ZPL}}=\frac{1}{\beta_{0}}\left(\frac{\tau_{0}}{\tau^{\prime}}-1\right)
$$

where $\beta_{0}$ is the Debye-Waller factor, recently estimated to be $2.55 \%$ [33], $\tau_{0}$ is the lifetime without the influence of the cavity, and $\tau^{\prime}$ is the reduced $\mathrm{N}-V$ center lifetime (see Appendix $\mathrm{C}$ for a derivation). In this paper, we choose the definition of the Purcell factor as the increased emission into the ZPL in the cavity only - rather than the increase in total emission - because it better reflects the coherent ZPL light that can be used for entanglement. A subset of the ZPL light, $F_{P}^{\mathrm{ZPL}} \beta_{0} /\left(F_{P}^{\mathrm{ZPL}} \beta_{0}+1\right)$, is collectable via the cavity mode; this definition means that $F_{P}^{Z \mathrm{PL}}=0$ without Purcell enhancement from the cavity.

To measure lifetime and fluorescence from $\mathrm{N}-V$ centers in the cavity under off-resonant excitation, we replace the measurement block in Fig. 1(d) with pulsed green excitation, pictured in Fig. 2(a). The cavity is again stabilized with a red laser and the excitation is provided by an $532 \mathrm{~nm}$

(a)

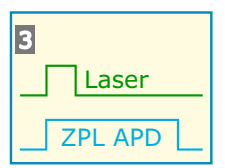

(b)

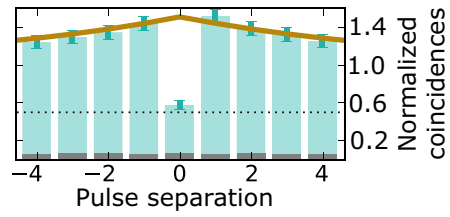

(c)

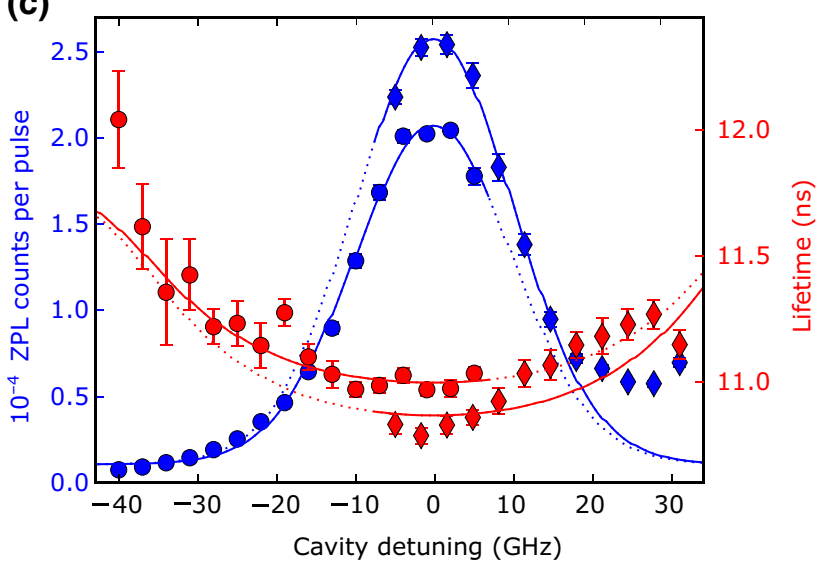

FIG. 2. Purcell enhancement of $\mathrm{N}-V$ centers under off-resonant excitation. (a) Measurement block for pulsed off-resonant excitation and detection in the ZPL. (b) Normalized pulsed autocorrelation measurement of $\mathrm{N}-V$ center fluorescence from the cavity on two ZPL detectors. Dark boxes on the bottom are background fluorescence between the pulses. We fit the data to a simple bunching model based on probabilistic state initialization into a bright state (solid line); see Appendix D 1. (c) Detuning sweep of the cavity with respect to the N- $V$ centers with measured ZPL fluorescence counts (blue) and lifetime (red). The data were taken on different days (circles and diamonds). We perform a joint fit of our model to both curves (solid lines); see Appendix D 1. The data in (b) and (c) are measured at different locations on the sample, which are also different from Fig. 1. 
picosecond pulsed laser input through the fiber. We collect the resulting fluorescence in the ZPL path on an avalanche photodiode (APD), and extract counts per pulse and lifetime as we sweep the detuning of the cavity. For further experimental details, see Appendix D 1.

In Fig. 2(c) we show one such detuning sweep, in which the fluorescence counts and lifetime vary with cavity detuning. The highest fluorescence counts and lowest $\mathrm{N}-\mathrm{V}$ center lifetime coincide, demonstrating Purcell-enhanced $\mathrm{N}-V$ center emission induced by coupling to the cavity. The widths of the fluorescence peak and of the lifetime reduction curve are several times broader than the cavity linewidth. To understand the quantitative behavior of the detuning sweep, we introduce a model that includes vibrations of the cavity and a spectral distribution of $\mathrm{N}-\mathrm{V}$ center transition frequencies (see Appendix C). By fitting this model to the data, we can extract the emission into the $\mathrm{ZPL}$ in the cavity, $F_{P}^{\mathrm{ZPL}} \beta_{0} /\left(F_{P}^{\mathrm{ZPL}} \beta_{0}+1\right)=(7.9 \pm 2.2) \%$, and the off-resonant lifetime, $\tau_{0}=11.8 \pm 0.2 \mathrm{~ns}$, which is consistent with the lifetime of $\mathrm{N}-V$ centers reported for bulk diamond $[47,48]$. The data were taken on two different days, leading to two different curves in Fig. 2(c) (circles and diamonds) that can be explained by drifts, which we account for in our modeling (Appendix D 1).

We investigate whether the emission is produced by a single emitter using an autocorrelation $\left(g^{(2)}\right)$ measurement. At most locations, there is little or no drop in coincidence counts at zero pulse separation. Therefore, we conclude that we are addressing several emitters within the cavity mode volume, likely because the high density of $\mathrm{N}-V$ centers in our sample lowers the chance of single center addressing with off-resonant excitation. In Fig. 2(b) we display the most significant drop in coincidences observed, which falls to $0.58 \pm 0.05$ at zero time delay $(0.54 \pm$ 0.05 with background correction). We observe significant bunching behavior for small pulse separations, which we attribute to probabilistic state initialization into a bright state (Appendix D 1). The enhancement of the ZPL by a factor of $3.9 \pm 0.9$ (assuming the same $\beta_{0}$ as in Ref. [33]) is a lower bound for the largest enhancement of single centers at this spot, because there are multiple $\mathrm{N}-V$ centers within the cavity mode volume.

\section{CONTINUOUS RESONANT EXCITATION}

Entanglement generation in quantum networking protocols requires coherent addressing of individual ZPL transitions with linewidths close to their lifetime-limited value [21]. To determine the suitability of our device for such tasks, we now move on to photoluminescence excitation (PLE) scans.

The measurement protocol for these scans is displayed in the inset of Fig. 3(a): short green pulses used for initialization of the $\mathrm{N}-V$ center in the negative charge and the $m_{s}=0$ spin state are interleaved with red measurement pulses. Fluorescence counts collected in the phonon sideband (PSB) during the red pulses are then correlated with simultaneously recorded wavemeter readings. This scheme thus allows predominant detection of the two $m_{s}=0$ spinconserving optical transitions per N- $V$ center; these transitions connect the ground state to the two optically excited states typically labeled as $E_{x}$ and $E_{y}$. For further details about this measurement sequence, see Appendix D 2.

A resulting PLE scan can be seen in Fig. 3(a). We observe a multitude of narrow lines per cavity spot, confirming our interpretation that there are multiple $\mathrm{N}-V$ centers present per cavity mode volume. In Fig. 3(b) we show an enlarged view into the red dashed region of Fig. 3(a); note that each binned point is comprised of many underlying individual data points. Importantly, the individual transition peaks can be spectrally distinguished, which is a prerequisite for single resonant $\mathrm{N}-V$ center addressing, as probed below.

To test the spectral stability of the transition peaks, we repeatedly scan the excitation laser over a $\mathrm{N}-V$ center ZPL transition. In Fig. 3(c) we show a series of 17 consecutive scans over the frequency region highlighted with purple dashed lines in Fig. 3(b). To correct for slow drifts in this measurement (likely due to temperature fluctuations in the cryostat), we fit a Gaussian lineshape to each individual PLE trace, and shift the lines to a common center frequency, displayed in Fig. 3(d); the resulting data are representative of the average spectral diffusion experienced on the timescale of one single frequency scan (largely induced by the continuous off-resonant repumping [37]). In Fig. 3(e) we show the averaged data of the original and centered scans in blue and red, respectively. From Gaussian fits to the data (solid lines), we extract full width at half maximum linewidths of $224 \pm 10$ and $190 \pm 9$ $\mathrm{MHz}$ for the original and centered cases, respectively. We probed the linewidths of $14 \mathrm{~N}-V$ center transitions during the course of this study, for a total of four different cavity positions. In Table I of Appendix D 2 we show the individual linewidths of each $\mathrm{N}-V$ center transition, which average to $204 \pm 59$ and $168 \pm 49 \mathrm{MHz}$ for the uncentered and centered cases, respectively. Thus, the linewidths in our sample are comparable to those measured in bulk diamond samples with similar protocols; close-to transform limited lines have been achieved without continuous green repumping in both bulk diamonds and in micron-thin membranes as used here [37,49]. Importantly, N- $V$ centers with this level of optical coherence have enabled previous entanglement generation experiments by exploiting resonant excitation for charge state and frequency preselection [21].

We next investigate the polarization behavior of $\mathrm{N}-V$ center transitions in the cavity by scanning the polarization angle of the excitation laser with a half wave plate (HWP), and observe a dependence of the background corrected fluorescence counts on this HWP angle [see 

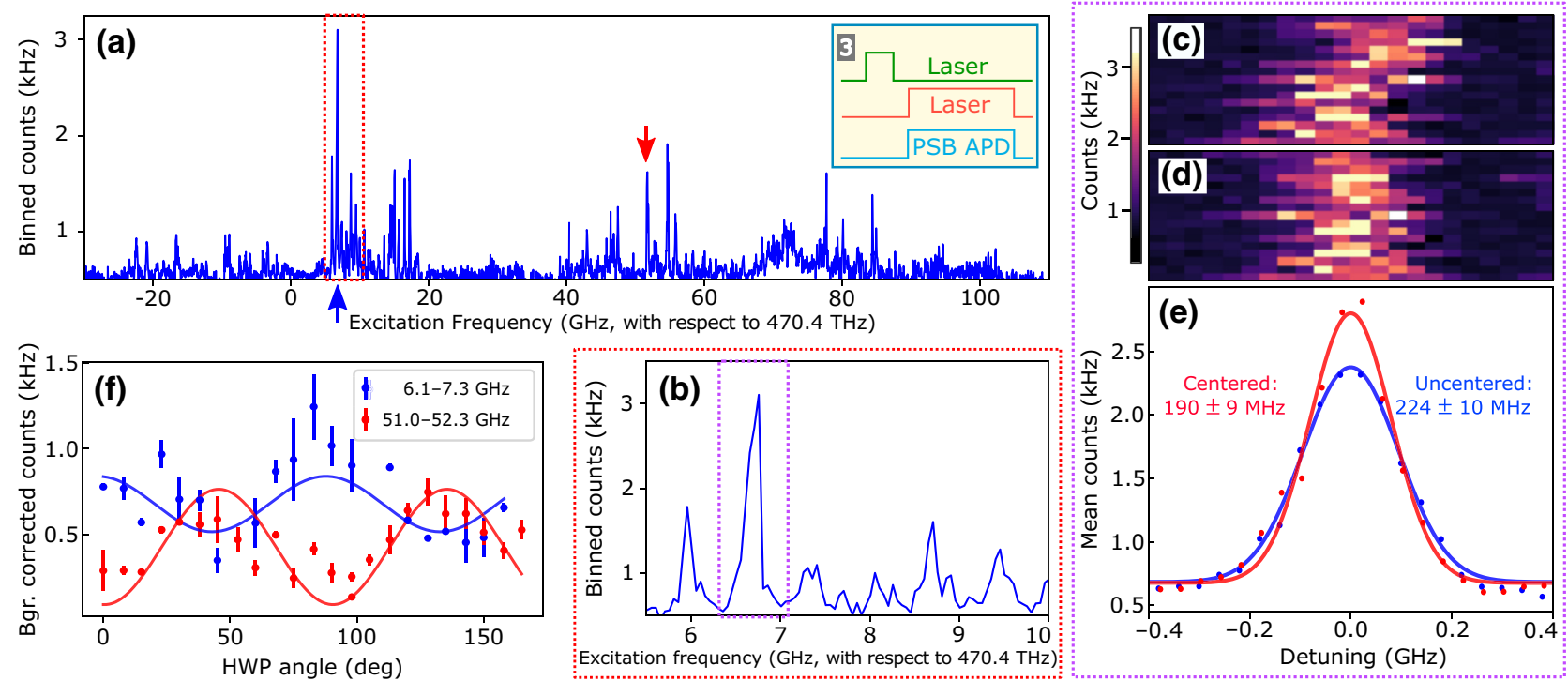

FIG. 3. Photoluminescence excitation (PLE) scans. (a) Broad PLE scan (cavity resonant with excitation laser, binned into $50 \mathrm{MHz}$ bins), revealing multiple bright lines per cavity position. Blue and red arrows indicate the center position of polarization angle scans displayed in (f). Inset: measurement block used during PLE scans. (b) Enlargement of the red dashed frequency region in (a). (c) Series of 17 consecutive PLE scans (measurement time approximately $15 \mathrm{~min}$ per trace) for the purple shaded frequency region in (b). (d) Same data as in (c), but center position of Gaussian fit to each individual trace used to correct for slow drift during the measurement. (e) Averaged and Gaussian fitted data of (c, blue) and (d, red), showing spectral diffusion limited linewidths of $224 \pm 10$ and $190 \pm$ $9 \mathrm{MHz}$, respectively. (f) Background corrected mean fluorescence counts during PLE scans for frequency regions of 6.1-7.3 GHz (blue) and $51.0-52.3 \mathrm{GHz}$ (red, each with respect to $470.4 \mathrm{THz}$ ) as a function of excitation laser half-wave plate (HWP) angle, showing that individual transitions are polarized. Solid lines are sinusoidal fits.

Fig. 3(f)]; polarization can be used to suppress resonant excitation pulses in a cross-polarization detection scheme [21], as demonstrated below. Interestingly, the N- $V$ centers in the two studied frequency clusters around 470.407 and $470.452 \mathrm{THz}$ show a different polarization behavior. A possible origin of this effect is a strain-induced splitting in transition frequencies for $E_{x}$ and $E_{y}$ polarized lines, as observed in Ref. [37]; future investigation is required to conclusively determine the origin.

\section{PULSED RESONANT EXCITATION}

Now that we can resolve individual $\mathrm{N}-V$ centers spectrally, we characterize their Purcell enhancement with a detuning sweep similar to that of Sec. III. The measurement block replaces off-resonant pulses with single frequency resonant pulses [Fig. 4(a)]. The sequence consists of a green repump pulse to initialize the $\mathrm{N}-V$ center predominantly in the negative charge and $m_{s}=0$ spin state, followed by a series of short (about $2 \mathrm{~ns}$ ) red pulses at the frequency of the $\mathrm{N}-V$ center transition. We record the fluorescence counts in the PSB path after each pulse, and extract the $\mathrm{N}-V$ center lifetime. For further details about the measurement sequence, see Appendix D 3.

First, we perform a pulsed autocorrelation measurement with two detectors in the PSB path to confirm that the individual peaks measured during photoluminescence excitation scans are indeed from single N- $V$ centers. In Fig. 4(b) we display a measurement from which we derive a normalized $g(2)$ value of $0.19 \pm 0.09(0.16 \pm 0.07$ with background correction) for zero pulse separation. Unlike the off-resonant case, the value at zero pulse difference clearly falls below 0.5 in all three $\mathrm{N}-V$ centers we tested, indicating that we are observing single quantum emitters.

For the same N- $V$ center measured in Figs. 3(c)-3(e), we sweep the detuning of the cavity from the $\mathrm{N}-V$ center transition while keeping the excitation pulses resonant with the latter to measure the Purcell enhancement. The collection efficiency of PSB emission is independent of the cavity length. However, the probability of exciting the $\mathrm{N}-V$ center depends on the overlap between the excitation laser frequency and the cavity resonance, so the PSB intensity should vary with detuning for fixed excitation power (see Appendix C). In the measurement [Fig. 4(c)], the fluorescence counts increase and the lifetime decreases when the cavity is on resonance with the $\mathrm{N}-V$ center, demonstrating that we observe Purcellenhanced $\mathrm{N}-V$ center emission. We fit our model to both curves and extract the fraction of ZPL emission into the collectable cavity mode, $F_{P}^{\mathrm{ZPL}} \beta_{0} /\left(F_{P}^{\mathrm{ZPL}} \beta_{0}+1\right)=(7.0 \pm$ $3.4) \%$ (see the definition in Sec. III), the off-resonant lifetime, $\tau_{0}=10.9 \pm 0.2 \mathrm{~ns}$, and the root-mean-squared cavity vibrations, $\sigma_{\text {vib }}=0.18 \pm 0.02 \mathrm{~nm}$ (see Appendix D 3). This Purcell factor corresponds to a cooperativity $C$ of 
(a)

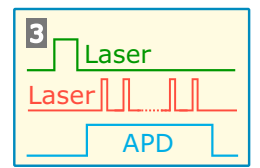

(b)

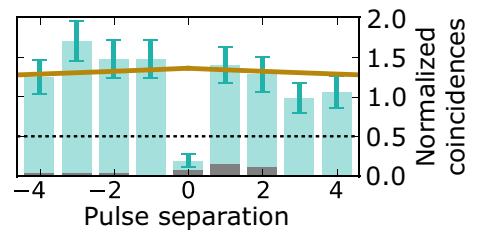

(c)

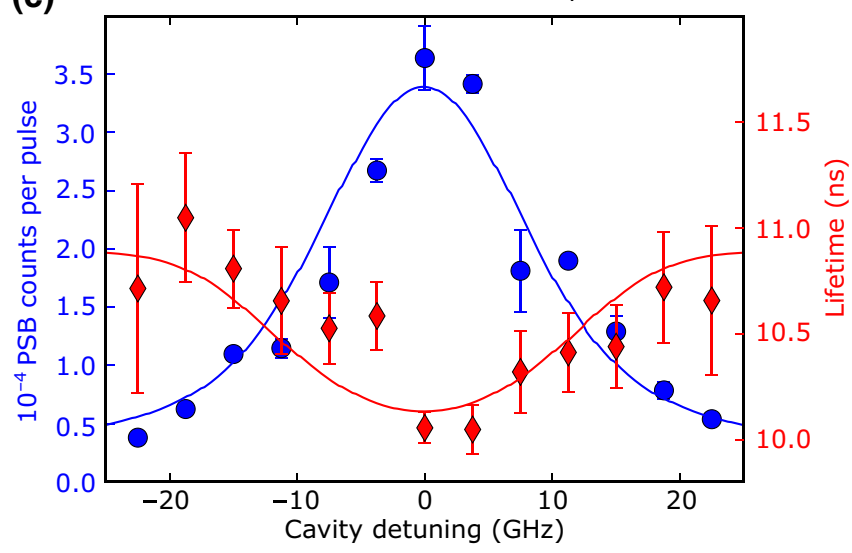

FIG. 4. Purcell enhancement of N- $V$ centers probed with resonant excitation. (a) Measurement block used for pulsed resonant excitation and detection in the PSB and ZPL. (b) Normalized pulsed autocorrelation measurement of $\mathrm{N}-V$ center fluorescence from the cavity on two PSB detectors. Dark boxes on the bottom are background fluorescence measured with the excitation detuned by $5 \mathrm{GHz}$. The fit accounts for bunching effects due to probabilistic state initialization into a bright state and the finite pulse train we apply (solid line); see Appendix D 3. (c) Detuning sweep of the cavity with respect to the $\mathrm{N}-V$ center with measured PSB fluorescence (blue) and lifetime (red). We perform a joint fit of both curves to a model (solid lines) with four free parameters (Appendix D 3). The data in (b) and (c) are measured on the same N- $V$ center as Figs. 3(c)-3(e), which is a different emitter than those studied in Figs. 2(b) and 2(c).

$0.08 \pm 0.04$ (see Appendix C). The enhancement of this $\mathrm{N}-V$ center is consistent with the enhancement we found for $\mathrm{N}-V$ centers under off-resonant excitation.

\section{ZPL COLLECTION AND FUTURE IMPROVEMENTS}

So far, we have only studied light emitted into the PSB after excitation with resonant light pulses. For quantum information applications, however, it is important to extract the emitted ZPL photons with high efficiency. In the current configuration, the excitation light is directly transmitted to the detector. Therefore, we separate out the ZPL photons from the bright excitation pulse with cross polarization and time-bin filtering [21,25], and shorten the excitation pulse further by introducing an additional electro-optic modulator (see Appendix A for details).

To be able to detect ZPL photons after a resonant excitation pulse, cross-polarization detection is especially important, because state-of-the-art photodetectors have a dead time longer than the lifetime of the $\mathrm{N}-V$ center; if a photon from the excitation pulse hits the detector, the dead time prevents detection of a ZPL photon, effectively reducing detection efficiency. Unfortunately, cross polarization only reduces the pulse power by a factor of 4 in our setup, which is likely due to vibrations of the freely hanging single-mode fiber in the cryostat. We additionally insert a $8.6 \mathrm{~dB}$ attenuation neutral-density (ND) filter into the ZPL path so that the efficiency of the detector remains high.

In Fig. 5(a) we display the fluorescence counts after a resonant excitation pulse, recorded simultaneously in the PSB and ZPL collection paths. The fluorescence in the ZPL path decays with a lifetime that agrees to within
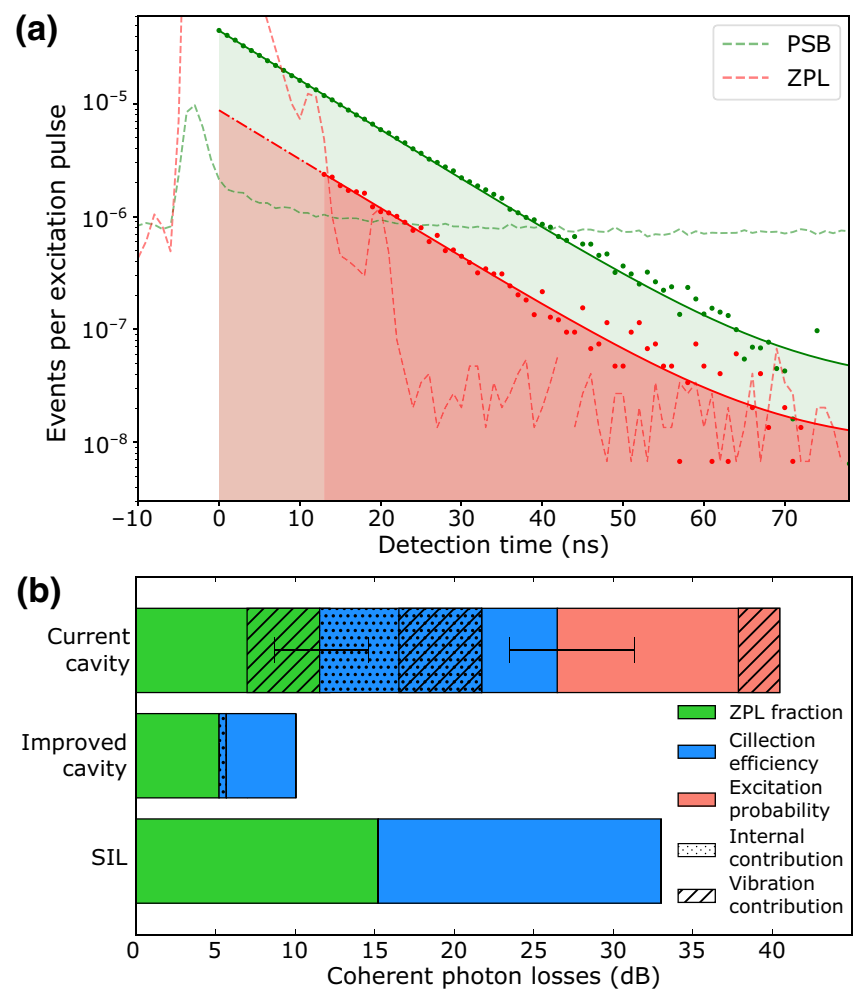

FIG. 5. ZPL and PSB fluorescence counts after resonant excitation and estimation of current and future ZPL count loss sources. (a) Background corrected PSB (green) and ZPL (red) fluorescence per excitation pulse (points), recorded at the same time. Background from the bright excitation pulse is shown with dashed lines (Appendix D 3). The parallel decay of an exponential fit to both curves (solid lines) indicates emission from the same $\mathrm{N}-V$ center. Shaded regions indicate areas for which fluorescence counts are summed to extract counts per excitation pulse (for the ZPL, we include the extrapolated region shaded in light red); see Appendix D 3. (b) Schematic of the sources of loss of coherent ZPL photons for the current cavity, the proposed improved cavity and a state-of-the art solid immersion lens (SIL) collection system. We breakdown the loss contributions into a ZPL fraction, a collection efficiency, and an excitation probability and separate the losses into vibration-induced losses (stripes) and internal cavity losses (dotted) (see Appendix F). 
error with the lifetime of the $\mathrm{N}-V$ center in the PSB path (Appendix D3). Thus, our technique enables us to see ZPL light from a $\mathrm{N}-V$ center in a cavity under resonant excitation.

Summing the fluorescence counts from the N- $V$ center in the ZPL path gives us a benchmark for the performance of our system as a collection enhancement tool. The excitation pulse obscures the initial counts, so we extrapolate a fit to the lifetime to extract the total $\mathrm{N}-V$ center counts in the ZPL and correct for the ND filter. We find $(9.3 \pm 0.2) \times 10^{-5}$ photons per pulse in the ZPL path and $(4.6 \pm 0.1) \times 10^{-4}$ in the PSB path. Because of low available laser power, we operate with both low initialization probability into the $m_{s}=0$ state and low excitation probability to the excited state (see Appendix F).

To better compare the cavity with other collection enhancement systems, and to understand current limitations and possible improvements in a future system, we break the loss of coherent photons down into its main contributions; see Fig. 5(b) (Appendix F contains a detailed derivation and full discussion of these values). For the current cavity system, about $14 \mathrm{~dB}$ of losses are associated with our limited probability of exciting the $\mathrm{N}-V$ center to its excited state from one resonant laser pulse, about $15 \mathrm{~dB}$ of losses result during photon collection, and about $12 \mathrm{~dB}$ of losses result from the $\mathrm{N}-V$ center emitting a photon in the PSB, rather than the ZPL.

Importantly, correcting for the excitation probability alone already raises the ZPL detection probability to about $2.0 \times 10^{-3}$, which is comparable to the collection of coherent ZPL photons achieved for a N- $V$ center in a solid immersion lens (about $5 \times 10^{-4}$ ) [24,25]. Thus, despite the relatively low collection efficiency from our current cavity, limited in large parts due to operation in the clipping regime of the fiber mirror, our system already produces ZPL photons at a level comparable to state-of-the-art noncavity systems (see Appendix F).

There are a number of changes that could improve ZPL collection under resonant excitation; we focus on three main developments that have already been achieved in other systems. First, introducing microwaves and a spin pumping laser is a standard technique for initializing and controlling the $\mathrm{N}-V^{-}$charge and $m_{s}=0$ spin state with high fidelity in bulk samples. The narrow spectral diffusion linewidths demonstrated in this work should make this possible in our system as well. Together with upgraded laser excitation pulse power, this should allow for near unity excitation per pulse, as is common for bulk diamond samples [50]. By increasing the polarization extinction by a factor of at least 100 by either fixing the fiber in the cryostat or switching to a polarization maintaining fiber, the excitation pulse can be suppressed sufficiently even for these higher excitation powers. Second, reducing the vibrations by a factor of 20 (from about 0.2 to about $0.01 \mathrm{~nm}$ ), as demonstrated in Refs. [14,51-54], would increase the ZPL detection fraction by a factor of about 16 . Our current data already show evidence for this potential improvement: by correlating the lifetime and the PSB fluorescence counts per resonant excitation pulse with the vibration level in the cryostat, we observe a reduction in lifetime (increase in fluorescence counts) from $10.02 \pm 0.12 \mathrm{~ns}[(2.5 \pm 0.1) \times$ $\left.10^{-4}\right]$ to $9.77 \pm 0.08 \mathrm{~ns}\left[(5.8 \pm 0.2) \times 10^{-4}\right]$ for data collected during high and low vibration time periods of the cryostat, respectively. We explore this correlation in detail in Appendix E. Finally, by working with a different fiber that is not clipping-loss limited, we expect to improve collection by a factor of 3 . A further advantage of the last two improvements is that the required laser power and polarization extinction are reduced by a factor of 5 and 25, respectively (see Appendix F). Together, these three improvements would raise the joint probability of producing and detecting a ZPL photon after short pulsed excitation to $10 \%$.

\section{CONCLUSION}

We demonstrate resonant excitation of cavity-coupled $\mathrm{N}-V$ centers with narrow optical transitions; the observed enhancement of collected coherent photons is in excellent agreement with our theoretical model. Using mutually nonexclusive numbers that have already each been achieved in several systems, we predict that we can increase the collected ZPL photons per excitation pulse to $10 \%$ in a future $\mathrm{N}-\mathrm{V}$-cavity system operated in a closedcycle cryostat. The demonstration of resonant excitation of a $\mathrm{N}-V$ center in a cavity represents a critical step on the path towards Purcell-enhanced remote entanglement generation.

Building on the previous success of $\mathrm{N}-V$ centers in entanglement generation and other network protocols [21, $23,24]$, a cavity enhanced $\mathrm{N}-V$-photon interface could dramatically improve entanglement rates and fidelity; single click and double click protocols would speed up by a factor of 100 and 10000 , respectively. This opens the door to experiments such as device-independent quantum key distribution and quantum repeaters $[55,56]$. In the longer run, we expect that the realization of fully coherent quantum emitters embedded in optical fiber-based cavities will enable more extensive quantum networks with long coherence times, a crucial step towards a quantum internet.

The data underlying the figures of this research article are available online [57].

\section{ACKNOWLEDGMENTS}

We thank Wouter Westerveld, Martin Eschen, Guus Evers, and Santi Sager La Ganga for experimental assistance, Thomas Fink for fabrication of the fiber mirror, Lennart van den Hengel for electron irradiation of the diamond, and Simon Baier and Conor Bradley for reviewing the manuscript. We acknowledge financial support 
from the EU Flagship on Quantum Technologies through the project Quantum Internet Alliance, the Netherlands Organisation for Scientific Research (NWO) through a VICI grant, the European Research Council (ERC) through an ERC Consolidator Grant, and the Royal Netherlands Academy of Arts and Sciences (KNAW) and Ammodo through an Ammodo KNAW Award.

\section{APPENDIX A: EXPERIMENTAL SETUP}

In Fig. 6 we show an overview of the experimental setup used in this work. The control electronics block [Fig. 6(a)] consists of a real-time controller (Jaeger Adwin Pro II), an arbitrary waveform generator (AWG, Tektronix 5014), a time-resolved photon counting module (Timetagger, Picoquant HydraHarp 400), and a PC, from which all measurements are controlled.

In the excitation path [Fig. 6(b)], $637 \mathrm{~nm}$ pulses with nanosecond time resolution are carved out of a red tuneable laser (Newfocus Velocity TLB-6300-LN) that is continuously monitored on a wavemeter (High Finesse WS6) using an amplitude electro-optic modulator (EOM, Jenoptik AM635) and an acousto-optic modulator (AOM, Gooch and Housego). After free space launching (FSC) of the beam, a polarizing beamsplitter (PBS, Thorlabs) maps polarization angle fluctuations into amplitude fluctuations. The red path is combined with directly modulated green laser light (515 nm, Cobolt MLD515) using a dichroic mirror (DM, Semrock FF560-FDi01), before the combined path is fiber coupled to the cavity. Half- (HWP) and quarter- (QWP) wave plates before and after the dichroic mirror correct for slow drift in polarization, and rotate the polarization of the resonant excitation light, respectively. For cavity linewidth measurements, we replace the amplitude modulating EOM with a phase modulating EOM (Jenoptik PM635, driven by a Rohde and Schwarz SGS100A signal generator), and connect it directly to the cavity fiber (IVG CU600). For measurements of the bandstructure of the cavity, we instead use a broadband "white light source" (NKT SuperK Compact) to illuminate the cavity fiber.

The position of the cavity fiber mirror can be tuned in situ via a high resonance frequency piezo positioning stage (JPE CPSHR1-a), mounted on top of a vibration isolator (JPE CVIP1) inside a closed-cycle cryostat operated at $T \sim 4 \mathrm{~K}$ (Montana C2); see Fig. 6(c). Light is collected from the cavity through a piezo-controlled (PI P-622/625) microscope objective [Mitutoyo Plan Apo 50X, numerical aperture (NA) 0.55]. We choose the flat mirror side to collect light from the cavity as it enables in principle unity coupling efficiency; the mode match between the beam in the fiber and the Gaussian mode in the cavity at the position of the fiber mirror leads to a finite coupling efficiency when collecting from the fiber side.

In the detection path [Fig. 6(d)], a 10:90 mirror mounted on a flip mount reflects light to a camera for visualization of the cavity modes. A dichroic mirror (Semrock DI 02-R365, pass edge at about $656 \mathrm{~nm}$ ) filters out green excitation light, before another flip mirror couples transmitted cavity light to a spectrometer (Princeton Instruments SP-2500i, used for bandstructure and rough N- $V$ center frequency estimation measurements) or an APD (Thorlabs APD130A2, used to measure the cavity linewidth). A dichroic mirror (Semrock DI 02-R365, pass edge at about $656 \mathrm{~nm}$ ) then transmits the $\mathrm{N}-V$ center ZPL light, while reflecting the PSB light. The PSB light then gets filtered (LPF, Semrock LP02-647RU-25) from any residual green excitation light, before being collected on APDs (LaserComponents or PicoQuant, detection with a multimode fiber, $50 \mu \mathrm{m}$ core diameter, $0.22 \mathrm{NA}$ ). A PBS (Thorlabs) in the ZPL filter path on a flip mirror filters out resonant excitation pulses. Before being collected (LaserComponents or PicoQuant, detection with a multimode fiber, $10 \mu \mathrm{m}$ core diameter, $0.1 \mathrm{NA}$ ), the ZPL light is filtered

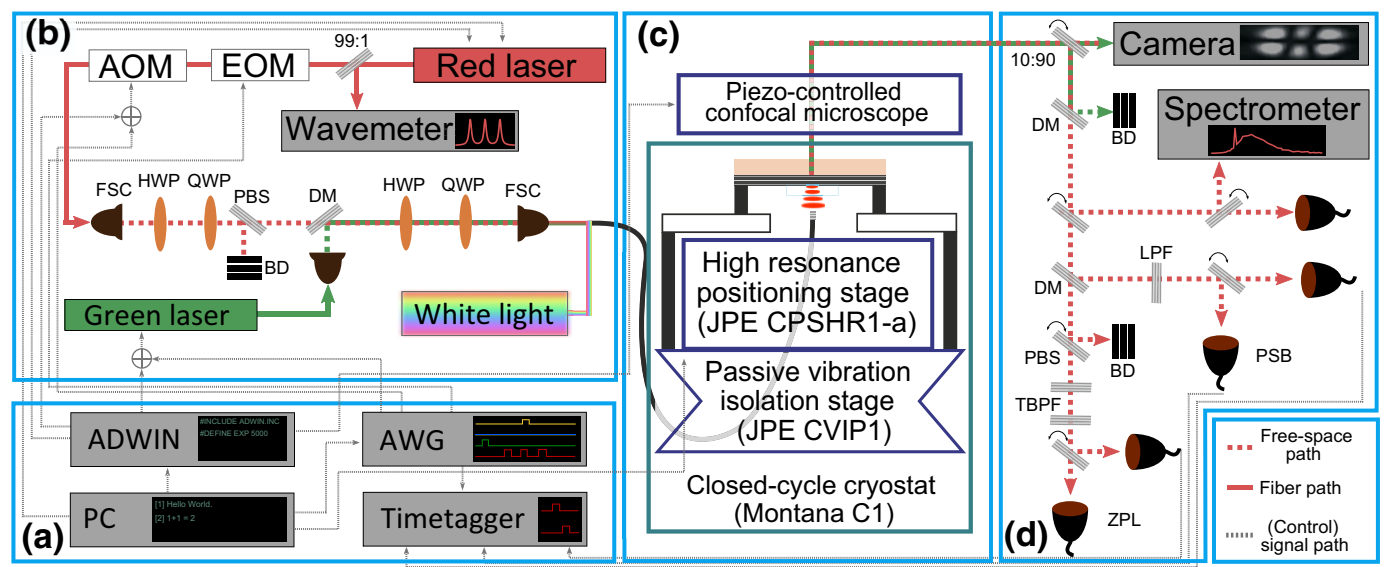

FIG. 6. Schematic of the experimental setup, consisting of the control electronics block (a), the excitation path (b), the cavity setup (c), and the detection path (d). See main text for definition of abbreviations. 
with two tuneable bandpass filters (TBPF, Semrock TBP 01-700/13, window $637 \pm 2 \mathrm{~nm}$ ) to suppress background fluorescence.

\section{APPENDIX B: CAVITY CHARACTERIZATION MEASUREMENTS}

To characterize the cavity, we first input white light through the fiber, and measure the cavity transmission on a spectrometer for different cavity lengths. In Fig. 7(a) we display the resulting dispersion diagram with avoided crossings between air and diamond modes [38,39]. From a fit to this data, we infer that we operate in an airlike mode with a diamond thickness of $5.8 \mu \mathrm{m}$ and a typical air gap between 5 and $7.5 \mu \mathrm{m}(7.3 \mu \mathrm{m}$ in the measurement shown) [38]. This gap cannot be reduced further, likely due to an angled mounting of, or dirt on, the fiber. To measure the cavity linewidth, we then scan the cavity length through a resonance and measure the corresponding transmission peak after the cavity with a photodiode (submillisecond timescale to minimize vibration contributions to the linewidth). We apply sidebands to the laser to calibrate the frequency of this scan. In the measurement displayed in Fig. 7(b), the linewidth of the cavity is $\kappa / 2 \pi=3.5 \pm 0.2 \mathrm{GHz}$, and the finesse is $2200 \pm 100$. Over the course of this work, we operated with a finesse between 1000 and 2500, dependent on the cavity position.

Based on the measured parameters of the cavity and a complete transfer matrix method described in Ref. [45], we can estimate the maximum possible Purcell enhancement in our cavity $\left(F_{P}^{\mathrm{ZPL}} \approx 6\right)$, which corresponds to a coupling between $\mathrm{N}-V$ centers and the cavity of $g / 2 \pi \approx 50 \mathrm{MHz}$. This estimate holds for a $\mathrm{N}-V$ center at the optimal depth location, which is a cavity standing wave antinode, and the optimal $x y$ position for maximum overlap with the Gaussian mode of the cavity. We describe the mismatch with the ideal position with a parameter, $\xi$, which we measure via the Purcell enhancement in the main text. The coupling is also reduced by vibrations, as discussed in Appendix C. The transition linewidth of the N- $V$ center was measured to be $\gamma / 2 \pi \approx 18.3_{-8.3}^{+5.7} \mathrm{MHz}$ in similar devices fabricated with the same method when accounting for power broadening [37]. In Secs. III and V, we measured the Purcell enhancement under off-resonant and resonant excitation and determined $F_{P}^{\mathrm{ZPL}} \approx 3$ and thus $g / 2 \pi \approx 35 \mathrm{MHz}$ [see Eqs. (C3) and (C4)]. The relative values of $g, \kappa$, and $\gamma$ put us in the weak coupling Purcell regime $(\gamma<g<\kappa)$, which is a favorable parameter range for collecting photons from an emitter [58].

\section{APPENDIX C: THEORETICAL MODEL}

\section{Purcell enhancement}

$\mathrm{N}-V$ centers exhibit limited emission into the ZPL, $\beta_{0}$, given by the Debye-Waller factor. Some states also couple (a)

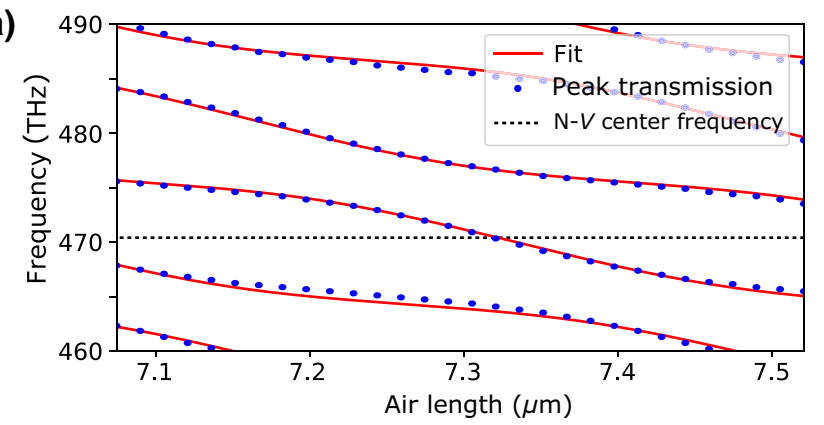

(b)

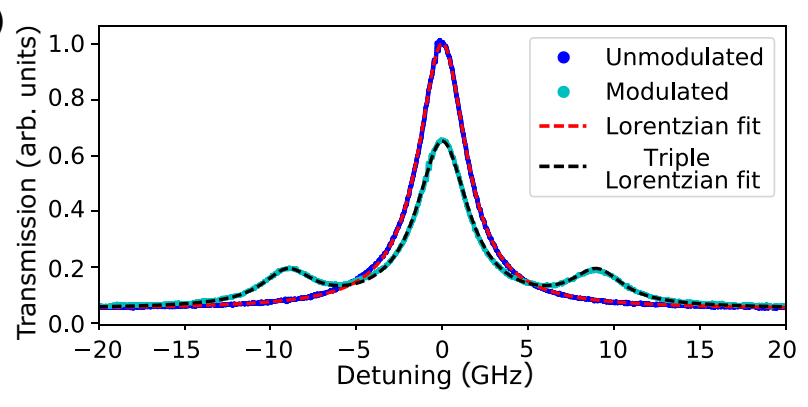

FIG. 7. Example data traces of cavity dispersion and finesse. (a) Cavity dispersion for the coupled membrane-air fiber-cavity system. The length of the cavity is swept and transmission is recorded on a spectrometer. From fits of the fundamental modes to a transfer matrix model, we can determine the diamond and air thicknesses to be 5.8 and $7.3 \mu \mathrm{m}$, respectively. (b) Finesse measurement. We scan the cavity quickly over a transmission peak to extract the cavity linewidth $(\kappa / 2 \pi=3.5 \pm 0.2 \mathrm{GHz})$ from fits to the data; the laser exciting the cavity is modulated with sidebands of $9 \mathrm{GHz}$ with an EOM, serving as frequency calibration reference.

to a nonradiative transition through an intersystem crossing [58]. Without interaction with a cavity, we can model the decay from the $\mathrm{N}-V$ center excited state, $\gamma_{0}$, with a radiative decay rate, $\gamma_{\text {rad }}$, and nonradiative decay rate, $\gamma_{\text {dark }}$, as

$$
\gamma_{0}=\beta_{0} \gamma_{\mathrm{rad}}+\left(1-\beta_{0}\right) \gamma_{\mathrm{rad}}+\gamma_{\text {dark }}
$$

Coupling to the mode of the cavity opens up another decay channel for the N- $V$ center, which we characterize with the cooperativity $C$ and the Purcell factor $F_{P}^{\mathrm{ZPL}}$. The decay rate from the $\mathrm{N}-V$ excited state, modified by the cavity, $\gamma^{\prime}$, is then given as

$$
\gamma^{\prime}=F_{P}^{\mathrm{ZPL}} \beta_{0} \gamma_{\mathrm{rad}}+\beta_{0} \gamma_{\mathrm{rad}}+\left(1-\beta_{0}\right) \gamma_{\mathrm{rad}}+\gamma_{\mathrm{dark}},
$$

with

$$
C=\frac{4 g^{2}}{\kappa \gamma}
$$

$$
F_{P}^{\mathrm{ZPL}}=\frac{C}{\beta_{0}},
$$


where $g / 2 \pi$ is the $\mathrm{N}-V$-cavity coupling, $\kappa / 2 \pi$ is the cavity linewidth, and $\gamma / 2 \pi$ is the previously measured optical transition linewidth of the $\mathrm{N}-V$ center [37,59]. We choose the definition of the Purcell factor as the enhancement of the ZPL-rather than the enhancement of the total emission from the $\mathrm{N}-V$ center-because it better reflects the increase in coherent light.

In this work, we primarily investigate transitions with linear polarization, and we preferentially initialize into the $m_{s}=0$ ground state with green excitation. The transitions in the $m_{s}=0$ manifold are $E_{x}$ and $E_{y}$, for which $\gamma_{\text {dark }}$ is much smaller than $\gamma_{\text {rad }}$ in bulk diamond $[48,60]$ : In Sec. V, we measure a reduced lifetime when the cavity is off resonance of $10.9 \pm 0.2 \mathrm{~ns}$, compared to the about $12 \mathrm{~ns}$ typically found in bulk diamond [48,60]; from this, we estimate that $\gamma_{\text {dark }} / \gamma_{\text {rad }} \approx 0.1$, so we assume that $\gamma_{\text {rad }} \gg \gamma_{\text {dark }}$, and simplify Eqs. (C1) and (C2) to

$$
\begin{aligned}
\frac{\gamma^{\prime}}{\gamma_{0}} & =\frac{\tau_{0}}{\tau^{\prime}} \\
& =1+F_{P}^{\mathrm{ZPL}} \beta_{0} \frac{\gamma_{\mathrm{rad}}}{\gamma_{\mathrm{rad}}+\gamma_{\mathrm{dark}}} \\
& \approx 1+F_{P}^{\mathrm{ZPL}} \beta_{0},
\end{aligned}
$$

where $\tau_{0}$ is the $\mathrm{N}-V$ center lifetime without the cavity and $\tau^{\prime}$ is the modified lifetime. The fraction emitted into the ZPL in the cavity mode is $F_{P}^{\mathrm{ZPL}} \beta_{0} /\left(F_{P}^{\mathrm{ZPL}} \beta_{0}+1\right)$. If the assumption that $\gamma_{\text {dark }}$ is negligible is incorrect then $F_{P}^{Z P L} \beta_{0}$ is increased by a factor of $\left(\gamma_{\text {rad }}+\gamma_{\text {dark }}\right) / \gamma_{\text {rad }}$. A simple rearrangement then gives us Eq. (1).

The rates of detected photons in the ZPL path, $C_{\mathrm{ZPL}}$, and the PSB path, $C_{\mathrm{PSB}}$, are

$$
\begin{aligned}
C_{\mathrm{ZPL}}(t) & =p_{\mathrm{ex}} \frac{F_{P}^{\mathrm{ZPL}} \beta_{0}}{F_{P}^{\mathrm{ZPL}} \beta_{0}+1} \eta_{\mathrm{ZPL}} \gamma^{\prime} e^{-\gamma^{\prime} t} \\
& =p_{\mathrm{ex}} F_{P}^{\mathrm{ZPL}} \beta_{0} \eta_{Z P L} \gamma_{0} e^{-\gamma^{\prime} t}
\end{aligned}
$$

and

$$
\begin{aligned}
C_{\mathrm{PSB}}(t) & =p_{\mathrm{ex}} \frac{1-\beta_{0}}{F_{P}^{\mathrm{ZPL}} \beta_{0}+1} \eta_{\mathrm{PSB}} \gamma^{\prime} e^{-\gamma^{\prime} t} \\
& =p_{\mathrm{ex}}\left(1-\beta_{0}\right) \eta_{\mathrm{PSB}} \gamma_{0} e^{-\gamma^{\prime} t},
\end{aligned}
$$

where $p_{\text {ex }}$ is the probability that a pulse excites the $\mathrm{N}-V$ center to its excited state, and $\eta_{\mathrm{ZPL}}\left(\eta_{\mathrm{PSB}}\right)$ is the detection efficiency for ZPL (PSB) photons from the cavity. As will be discussed in Appendix F2, it is helpful to break the ZPL detection efficiency down into internal and external efficiencies with $\eta_{\mathrm{ZPL}}=\eta_{\text {int }} \eta_{\text {ext }}$. A number of these parameters depend on the normalized intracavity power $P$ that itself depends on the detuning between the cavity and the transition, $\Delta$ :

$$
P(\Delta)=\frac{\kappa^{2} / 4}{\kappa^{2} / 4+\Delta^{2}} .
$$

This modifies $F_{P}^{\mathrm{ZPL}}$ and $\eta_{\mathrm{ZPL}}$ to

$$
\begin{gathered}
F_{P}^{\mathrm{ZPL}}(\Delta)=F_{P}^{\mathrm{ZPL}}(0) P(\Delta), \\
\eta_{\mathrm{ZPL}}(\Delta)=\eta_{\mathrm{ext}} \eta_{\mathrm{int}}(0) P(\Delta) .
\end{gathered}
$$

The PSB collection is not resonant with the cavity, and its spectral distribution lies mostly outside of the stopband of the mirror, so there should be minimal changes from small variations in cavity detuning. For the same reason, green excitation light is also independent of the cavity detuning. However, resonant red excitation depends strongly on cavity detuning, and the corresponding power in the cavity. The Rabi frequency is proportional to the square root of the intracavity power that scales with $P(\Delta)$. Therefore, in the resonant excitation case

$$
p_{\mathrm{ex}}(\Delta)=\sin \left[\phi_{p}(0) \sqrt{P(\Delta)} / 2\right]
$$

Here $\phi_{p}(0)$ is the Rabi rotation angle induced by the pulse on resonance (e.g., $\pi$ for a complete population of the excited state). In the weak excitation limit explored in this work, this can be approximated as $\phi_{p}(0) \sqrt{P(\Delta)} / 2$.

\section{Vibration model}

The above simple model does not take into account the vibrations in the cavity length. We extend the model of Ref. [45] to build a complete numerical transfer matrix model for the cavity. We assume a Gaussian distribution of cavity lengths, $f_{\mathrm{vib}}$, given as

$$
f_{\mathrm{vib}}(d L)=\frac{1}{\sqrt{2 \pi \sigma^{2}}} e^{-d L^{2} /\left(2 \sigma^{2}\right)},
$$

where $\sigma$ is the width of the length distribution, and $d L$ is the length fluctuations around a certain cavity length, as induced from vibrations. We then find the Purcellenhanced fraction of emission into the ZPL $\left[F_{p}(d L) \beta_{0}\right]$ for each $d L$.

\section{a. Off-resonant excitation}

Under off-resonant excitation, the probability of exciting the $\mathrm{N}-V$ center does not depend on the length of the cavity. For each frequency detuning, $\Delta$, we find the equivalent cavity detuning length, $L_{\text {det }}$, using our complete model of the cavity dispersion relationship. We integrate Eq. (C6) 
over the length distribution in Eq. (C12) to determine the emitted counts in the ZPL:

$$
\begin{aligned}
C_{\mathrm{ZPL}}( & \left.t, L_{\mathrm{det}}\right) \\
= & p_{\mathrm{ex}} \gamma_{0} \int d L f_{\mathrm{vib}}(d L) g_{\mathrm{nv}}\left(L_{\mathrm{det}}+d L\right) \beta_{0} F_{P}^{\mathrm{ZPL}}\left(L_{\mathrm{det}}+d L\right) \\
& \times \eta_{\mathrm{ZPL}}\left(L_{\mathrm{det}}+d L\right) \exp \left[-\gamma^{\prime}\left(L_{\mathrm{det}}+d L\right) t\right] \\
= & p_{\mathrm{ex}} \beta_{0} F_{P}^{\mathrm{ZPL}}(0) \eta_{\mathrm{ext}} \eta_{\mathrm{int}}(0) \gamma_{0} \\
& \times \int d L f_{\mathrm{vib}}(d L) g_{\mathrm{nv}}\left(L_{\mathrm{det}}+d L\right) P\left(L_{\mathrm{det}}+d L\right)^{2} \\
& \times \exp \left[-\left\{1+\beta_{0} F_{P}^{\mathrm{ZPL}}\left(L_{\mathrm{det}}+d L\right)\right\} \gamma_{0} t\right] .
\end{aligned}
$$

We then numerically integrate and fit the function $C(t)$ to an exponential decay to determine the measured lifetime $\tau(\Delta)$. We also sum over $t$ to find total counts $C_{\text {tot }}(\Delta)$. For the case of multiple N- $V$ centers, we also introduce an extra Gaussian broadening term, $g_{\text {nv }}$, inside the integral. The exact form of $g_{\mathrm{nv}}$ depends on the (unknown) distribution of $\mathrm{N}-V$ centers, but we find that the Purcell factor we extract is relatively insensitive to the shape we pick.

\section{b. Resonant excitation}

Under resonant excitation, the probability of exciting the $\mathrm{N}-V$ center depends strongly on the excitation laser detuning from the cavity. This modifies the function for counts from the cavity slightly. We integrate the count curves in the same way as in the case of off-resonant excitation to determine the total counts. For collection from the ZPL and the PSB, respectively, we get

$$
\begin{aligned}
C_{\mathrm{ZPL}}\left(t, L_{\mathrm{det}}\right)= & \frac{p_{\mathrm{in}} \phi_{p}(0)}{2} \beta_{0} F_{P}^{\mathrm{ZPL}}(0) \eta_{\mathrm{ext}} \eta_{\mathrm{int}}(0) \gamma_{0} \\
& \times \int d L f_{\mathrm{vib}}(d L) P\left(L_{\mathrm{det}}+d L\right)^{5 / 2} \\
& \times \exp \left[-\left\{1+\beta_{0} F_{P}^{\mathrm{ZPL}}\left(L_{\mathrm{det}}+d L\right)\right\} \gamma_{0} t\right],
\end{aligned}
$$

$$
\begin{aligned}
C_{\mathrm{PSB}}\left(t, L_{\mathrm{det}}\right)= & \frac{p_{\text {in }} \phi_{p}(0)}{2}\left(1-\beta_{0}\right) \eta_{\mathrm{PSB}} \gamma_{0} \\
& \times \int d L f_{\mathrm{vib}}(d L) \sqrt{P\left(L_{\mathrm{det}}+d L\right)} \\
& \times \exp \left[-\left\{1+\beta_{0} F_{P}^{\mathrm{ZPL}}\left(L_{\mathrm{det}}+d L\right)\right\} \gamma_{0} t\right],
\end{aligned}
$$

where $p_{\text {in }}$ is the probability of initializing in the $m_{s}=0$ state. We do not include $g_{\text {nv }}$ because we only excite single $\mathrm{N}-V$ centers in the case of resonant excitation. Counts and lifetime are then calculated in the same manner as for the off-resonant case.

\section{APPENDIX D: MEASUREMENT TECHNIQUES AND DATA ANALYSIS}

\section{Off-resonant excitation}

\section{a. Protocol and timings}

For the off-resonant excitation measurements described in Sec. III, a measurement sequence starts by stabilizing a red excitation laser to a wavemeter, followed by stabilization of the cavity on transmission to that frequency. We excite the cavity with an approximate 65 picosecond pulsed green laser (Onefive Katana, Advanced Laser Diode Systems PiLas, $532 \mathrm{~nm}$, repetition rate $1 \mathrm{MHz}$, continuous output power measured after the cavity about 1 $\mathrm{mW}$ ), and collect resulting fluorescence in the ZPL with an APD and a time-resolved photon counting module [see Fig. 2(a)]. Because of a favorable signal-to-noise ratio, we choose to collect light in the ZPL, rather than in the PSB, as off-resonant excitation results in increased background in our sample. We stabilize the cavity on a certain detuning frequency point (always on $\mathrm{N}-V$ center resonance in case of a $g^{(2)}$ measurement) about every $10 \mathrm{~s}$ to mitigate slow drifts during the measurement.

We first take a background trace for a detuning of about $100 \mathrm{GHz}$ below the $\mathrm{N}-V$ center resonance frequency, so that we can be sure to not record any $\mathrm{N}-V$ center light; as the background is spectrally flat in the case of offresonant excitation, we can then use the data to correct for background fluorescence in the subsequent measurement sequence (background lifetime $6.6 \pm 0.6 \mathrm{~ns}$ ). We then start a measurement, which consists of 16 (14) repeated scans over the full detuning in the case of the diamond-shape (circular-shape) marked data in Fig. 2(c), each of which is comprised of 12 (16) frequency steps that take about 6 min each. The data were taken on two different days, with slight drifts in cavity position (and thus $\mathrm{N}-V$-cavity coupling) in between. Because the cavity filters out the signal off resonance, there is a limit to how far we can detune and accurately measure a lifetime, so we do not include data beyond that point. For Fig. 2(c), we include data with a signal-to-noise ratio of about 20 (cavity on resonance with the $\mathrm{N}-V$ center) down to about 5 (point of largest detuning between cavity and $\mathrm{N}-V$ frequency).

\section{b. Detuning sweep analysis and additional data}

An example time trace, corresponding to the underlying data for the frequency detuning point of $2 \mathrm{GHz}$ from the $\mathrm{N}-V$ center frequency [diamond-shaped data, Fig. 2(c)] can be seen in Fig. 8(a). To fit the full data of Fig. 2(c) to our theoretical model [Eq. (C13)], we proceed in the following way. We first perform a joint fit with five parameters (N- $V$ center frequency, on-resonance counts, root-meansquared vibrations level, off-resonance $\mathrm{N}-V$ center lifetime, and mismatch with the cavity) to all data. A sixth parameter, the Gaussian distribution of $\mathrm{N}-V$ center transition 

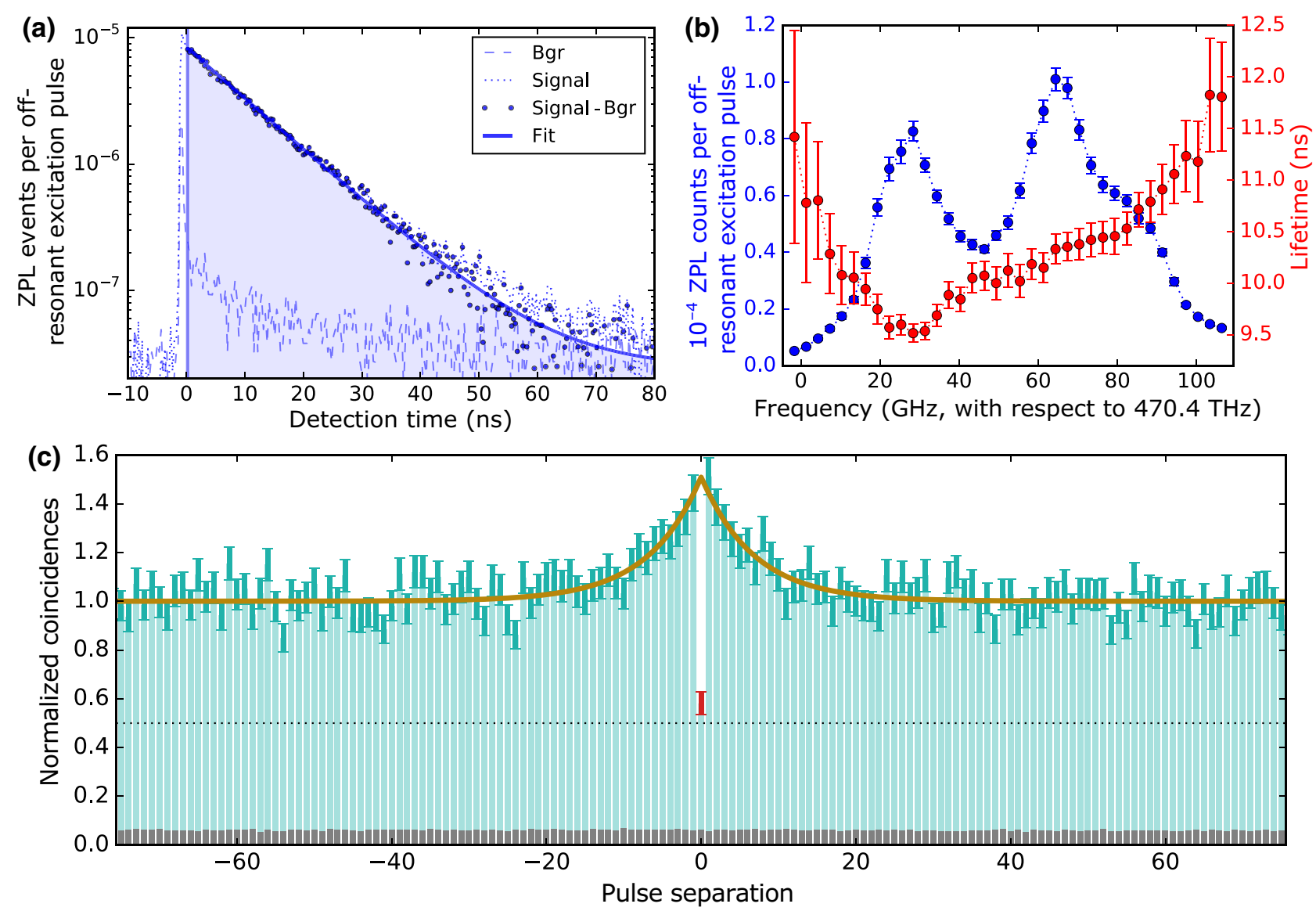

FIG. 8. Example off-resonant excitation time trace, additional detuning sweep, and full photon autocorrelation data. (a) Example trace of the data taken during off-resonant excitation. From a fit to the background corrected data, we extract a lifetime of $10.71 \pm 0.08$ $\mathrm{ns}$, and $(2.6 \pm 0.1) \times 10^{-4} \mathrm{ZPL}$ detection events per off-resonant excitation pulse (blue shaded area). (b) Detuning sweep of the cavity with respect to the $\mathrm{N}-V$ centers with extracted ZPL fluorescence counts (blue) and lifetime (red). This measurement is analogous to that displayed in Fig. 2(c), but taken at a different cavity position. (c) Full data for the pulsed photon autocorrelation measurement displayed in Fig. 2(b), with a total measurement time of approximately $65 \mathrm{~min}$. Gray bars represent background counts between the pulses, green bars represent integrated counts per pulse, and a red point represents the extracted value for zero pulse separation between detector clicks. The solid brown line is a fit to a simple bunching model based on probabilistic state initialization into a bright state. The dashed black line at 0.5 normalized coincidences represents the theoretical boundary for single emitter emission.

frequencies (as we are looking at multiple $\mathrm{N}-V$ centers per cavity spot) is kept fixed, as it is interchangeable with the vibration level; we choose a value that is consistent with typical photoluminescence excitation results (we also interchanged this width parameter to a square rather than a Gaussian distribution, leading to similar results). We then extract the Purcell enhancement and lifetime parameters reported in the main text from this overall fit. In a second step, we fix the $\mathrm{N}-V$ center frequency, vibrations level, and off-resonant lifetime, and refit the two traces (taken on several days) individually, allowing different on-resonance counts and cavity mismatch values. We use the results of these fits to plot the solid lines displayed in Fig. 2(c).

An additional detuning sweep, measured on a different cavity position, can be seen in Fig. 8(b). The shape of the lifetime and count curves indicates that, while the N$V$ centers are clustered around two frequency values, there are potentially many more $\mathrm{N}-V$ center frequencies around those two values, making it hard to fit our full model to the data. The location of the two peaks is consistent with the data reported in Fig. 1(d) (green and red curves). Since the green off-resonant laser both excites the $\mathrm{N}-V$ centers and initializes them predominantly in the negative charge and $m_{s}=0$ spin state, we expect the measured counts to be dominated from $m_{s}=0$ spin-conserving optical transitions, typically labeled as $E_{x}$ and $E_{y}$. A slight strain in the sample, as also observed in Ref. [37], seems to be a likely cause for the origin of the splitting between the peaks. Future investigation beyond the current work is required to confirm the mechanism behind this effect. 


\section{c. Photon autocorrelation analysis}

The data underlying the photon autocorrelation measurement displayed in Fig. 2(b) can be seen in Fig. 8(c). We compute the time differences between ZPL clicks after splitting the signal and measuring with two photodetectors, and integrate the counts for the time differences corresponding to a certain pulse separation number. We then fit a simple bunching model based on a three-level system to the resulting data [61], as the green laser leads to a finite state initialization into a bright state. We find a steady-state initialization probability of $0.66 \pm 0.06$, which is consistent with the literature $[62,63]$. From this fit, we can extract that the normalized autocorrelation falls to $0.58 \pm 0.05$ for zero pulse separation, or $0.54 \pm 0.05$ with background correction. The $g^{(2)}$ data displayed here represent the deepest dip we found. We therefore conclude that the N- $V$ center emission is predominantly from multiple $\mathrm{N}-V$ centers per cavity spot in the off-resonant excitation case.

\section{Photoluminescence excitation scans}

\section{a. Protocol and timings}

For the PLE scans described in Sec. IV, a measurement sequence starts by stabilizing a red excitation laser to a wavemeter, followed by stabilization of the cavity on transmission to that frequency. The measurement block, depicted in the inset of Fig. 3(a), then consists of short green pulses $(5 \mu \mathrm{s}, 60 \mu \mathrm{W})$ used for initialization of the $\mathrm{N}-V$ center in the negative charge and $m_{s}=0$ spin state, which are interleaved with red measurement pulses (75 $\mu \mathrm{s}, 20 \mathrm{nW}$ ); counts collected in the PSB during these red pulses are then correlated with simultaneously recorded wavemeter readings. To prevent background from building up, we introduce a wait time between a green and red pulse of $0.5 \mathrm{~ms}$, and break the red pulse down into 250 equal pulses of $300 \mathrm{~ns}$ each, with a wait time of $6.5 \mu \mathrm{s}$ in between red pulses. We sweep the excitation laser frequency after 250 green pulse repetitions in steps of about $2 \mathrm{MHz}$, and stabilize the cavity about every $20 \mathrm{~s}$ to the excitation laser.

\section{b. Additional spectral stability data}

The spectral stability of all tested $\mathrm{N}-V$ center transitions is displayed in Table I. We extract the data following two different approaches: the first is to directly average the counts from all PLE scan repetitions on a certain transition, leading to a value that includes slow drifts (several minutes-hours timescale), which we refer to as the "Gaussian linewidth." In a second method, we take out small drifts over the course of the measurement (possibly induced by temperature fluctuations in the cryostat leading to slight variation in membrane strain [and thus resonance position]). We do this by fitting each individual scan first, reshifting all scans corresponding to a certain transition
TABLE I. Spectral stability data for all measured N- $V$ center transitions. We extract an average Gaussian linewidth of $204 \pm 59 \mathrm{MHz}$ and a centered linewidth of $168 \pm 49 \mathrm{MHz}$. For a definition of these quantities, see the text. Transition 7 corresponds to the transition probed in Figs. 3(c)-3(e).

\begin{tabular}{lcccc}
\hline \hline $\begin{array}{l}\text { Transition } \\
\text { number }\end{array}$ & $\begin{array}{c}\text { Cavity } \\
\text { area } \\
\text { number }\end{array}$ & $\begin{array}{c}\text { Center position } \\
(\mathrm{GHz}, \text { with respect } \\
\text { to 470.4 THz) }\end{array}$ & $\begin{array}{c}\text { Gaussian } \\
\text { linewidth } \\
\text { (MHz) }\end{array}$ & $\begin{array}{c}\text { Centered } \\
\text { linewidth } \\
\text { (MHz) }\end{array}$ \\
\hline 1 & 1 & 16.3 & $105 \pm 9$ & $100 \pm 6$ \\
2 & 1 & 17.6 & $154 \pm 11$ & $125 \pm 6$ \\
3 & 1 & 20.0 & $146 \pm 5$ & $131 \pm 8$ \\
4 & 1 & 24.7 & $161 \pm 11$ & $110 \pm 6$ \\
5 & 2 & 18.6 & $187 \pm 18$ & $192 \pm 22$ \\
6 & 3 & 12.3 & $175 \pm 16$ & $175 \pm 16$ \\
7 & 4 & 6.7 & $224 \pm 10$ & $190 \pm 9$ \\
8 & 4 & 8.2 & $264 \pm 11$ & $251 \pm 13$ \\
9 & 4 & 17.8 & $273 \pm 41$ & $161 \pm 17$ \\
10 & 4 & 19.3 & $207 \pm 25$ & $136 \pm 26$ \\
11 & 4 & 47.8 & $194 \pm 18$ & $143 \pm 7$ \\
12 & 4 & 48.6 & $217 \pm 19$ & $175 \pm 9$ \\
13 & 4 & 50.9 & $210 \pm 16$ & $201 \pm 12$ \\
14 & 4 & 69.4 & $333 \pm 56$ & $257 \pm 54$ \\
Mean & & & $204 \pm 59$ & $168 \pm 49$ \\
\hline \hline
\end{tabular}

with respect to a common mean frequency, and then extracting the "centered linewidth" from a fit to the averaged shifted data (minutes timescale). The second value still includes spectral diffusion and thus follows a Gaussian distribution, as we constantly interleave green pulses that reshuffle the $\mathrm{N}-V$ center charge environment with red measurement pulses. The Gaussian linewidth averages to $204 \pm 59 \mathrm{MHz}$, while the centered linewidth averages to $168 \pm 49 \mathrm{MHz}$ over all $\mathrm{N}-V$ center transitions investigated in this study; these values are consistent with previous findings on a sample fabricated using the same process flow [37]. Importantly, they are also consistent with values extracted for bulk diamond samples under off-resonant repumping, and $\mathrm{N}-V$ centers with similar optical coherence have been used in previous entanglement generation protocols [21].

\section{Resonant excitation}

\section{a. Protocol and timings}

For the resonant excitation measurements described in Secs. V and VI, a measurement sequence starts by stabilizing a red excitation laser to a wavemeter, followed by stabilization of the cavity on transmission to that frequency. This sets the cavity length to a certain detuning point from a $\mathrm{N}-V$ center transition frequency that we precharacterize using photoluminescence excitation scans. The red excitation laser is then stabilized to this $\mathrm{N}-V$ center transition frequency using the wavemeter a second time. The measurement block, which starts next and is depicted in the inset of Fig. 3(a), consists of short green pulses (10 
$\mu \mathrm{s}, 60 \mu \mathrm{W})$ used for initialization of the $\mathrm{N}-V$ center in the negative charge and $m_{s}=0$ spin state, that are interleaved with short red measurement pulses (nominal time $2 \mathrm{~ns}$, generated by a cascade of two electro-optic modulators and one acousto-optic modulator and an AWG; see Appendix A); counts collected in the PSB or ZPL in a time window around these resonant excitation pulses are then recorded with a time-resolved photon counting module. To prevent background from building up, we introduce a wait time between a green and a red pulse of $1.8 \mathrm{~ms}$, and break the red pulse down into 500 equal pulses, with a wait time of $6.5 \mu \mathrm{s}$ in between red pulses. The cavity is stabilized about every $20 \mathrm{~s}$ to the desired cavity detuning point, after which the laser is stabilized to the $\mathrm{N}-V$ center transition frequency again, and a new measurement block is started. We repeat measurements for a certain cavity detuning point for about $15 \mathrm{~min}$, before we move on to measure a background file for the same amount of time. For the background measurement block, we subtract a certain frequency value from both the cavity stabilization frequency and the $\mathrm{N}-V$ center excitation frequency, for which we check independently that no N- $V$ center transition is close by. Before starting a measurement on the next detuning point, we interleave several PLE scans, and extract the current $\mathrm{N}-V$ center transition frequency in an automated fashion, to account for slow drifts.

\section{b. Data analysis}

An example time trace, corresponding to the underlying data of Fig. 5(a), can be seen in Fig. 9(a) for the ZPL data after resonant excitation, and in Fig. 9(b) for the PSB data after resonant excitation. The data are taken while keeping the detuning between the cavity and $\mathrm{N}-V$ center transition frequency at zero, and are an average over 16 experimental repetitions (about $4 \mathrm{~h}$ of data). As discussed in Sec. VI, (a)
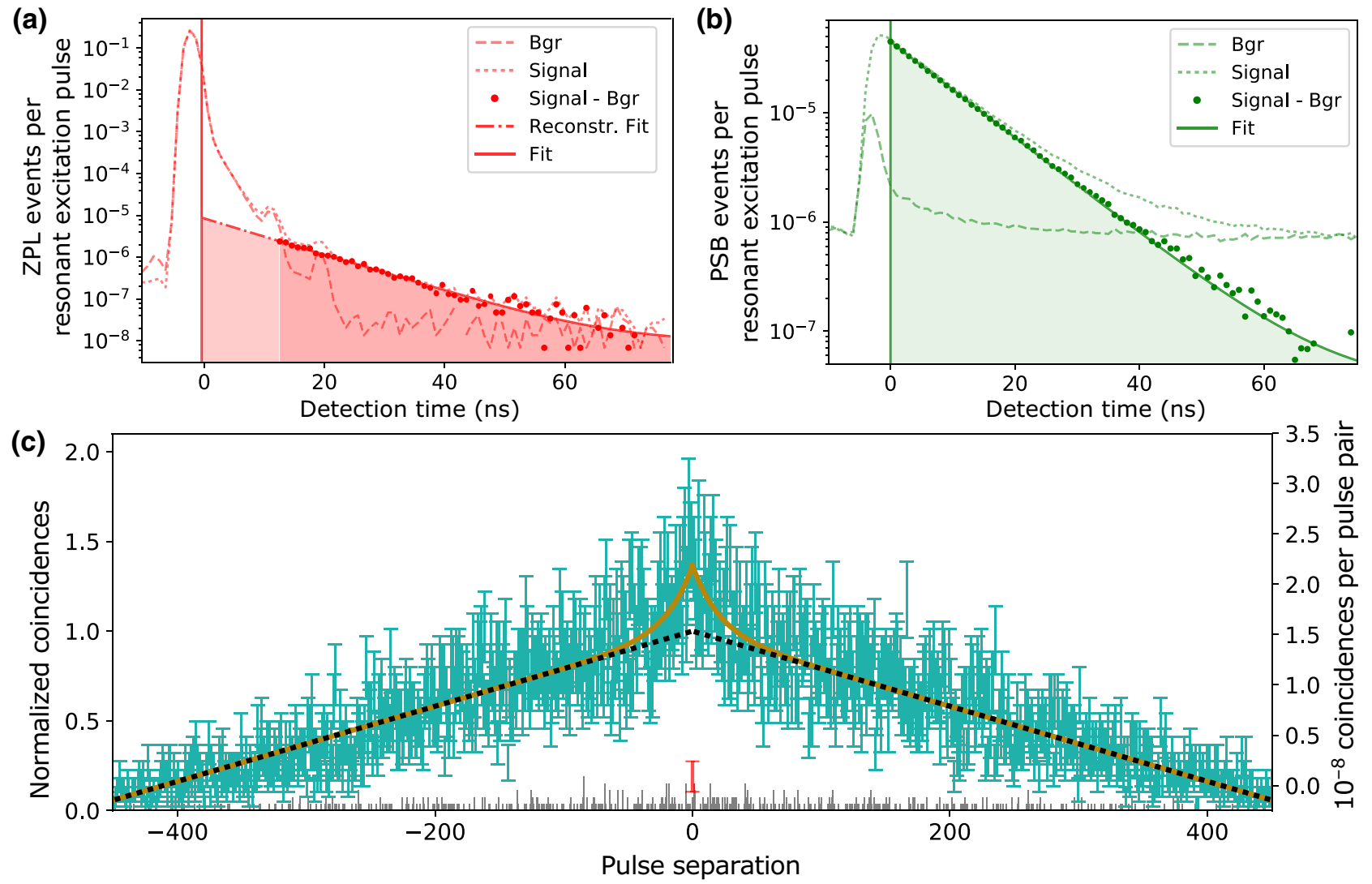

FIG. 9. Resonant excitation example data traces and full photon autocorrelation data. Example trace of ZPL (a) and PSB (b) detection events after a resonant excitation pulse, overlaid with a fit to the data [the same data as used in Fig. 5(a)]. The shaded area indicates the regions used to extract total counts per excitation pulse values. (c) Full data for the pulsed photon autocorrelation measurement displayed in Fig. 4(b), with a total measurement time of approximately $6 \mathrm{~h}$. Gray bars represent background counts extracted from independent measurements (see the text), green bars represent integrated and normalized counts per pulse, and a red point represents the extracted value for zero pulse separation. The solid brown line is a fit to a simple bunching model based on probabilistic state initialization into a bright state. The dashed black line represents the normalization line extracted from a fit that is a sloped line due to a finite number of excitation pulses per pulse train (see the text).

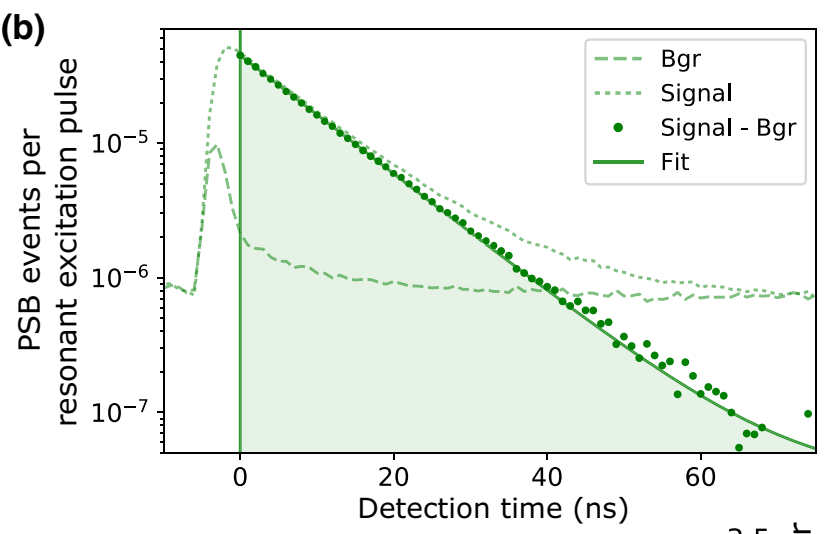


cross polarization only suppresses the pulse by a factor of about 4 in our system, likely due to vibrations of the freely hanging single-mode fiber in the cryostat causing polarization fluctuations. Therefore, we opted to use a $8.6 \mathrm{~dB}$ neutral density filter in the ZPL path to reduce detector blinding.

From exponential fits to the ZPL and PSB data, we extract lifetimes of $9.97 \pm 0.22$ and $9.87 \pm 0.02 \mathrm{~ns}$, respectively. Since the data are taken simultaneously in the ZPL and PSB, and agree within error bars, we conclude that our technique enables us to resolve resonant, coherent $\mathrm{N}-V$ center photons after a resonant excitation pulse. By integrating the counts in the red and green shaded areas in Figs. 9(a) and 9(b), we extract photon collections of $(9.3 \pm 0.2) \times 10^{-5} \mathrm{ZPL}$ photons and $(4.6 \pm 0.1) \times 10^{-4}$ PSB photons per excitation pulse, respectively.

To fit the full data of Fig. 4(c) to our theoretical model [Eq. (C15)], we proceed in the following way. We first fit the curve of PSB counts per resonant excitation pulse with the maximum counts and root-mean-squared vibration level as free parameters. We use the vibration level determined from this first fit as a fixed value for a second fit, in which we fit the lifetime curve with the $\mathrm{N}-V$-cavity mismatch and $\mathrm{N}-V$ off-resonant lifetime as free parameters. From the results of this fit, we then calculate the Purcell enhancement, and plot the model displayed with solid lines in Fig. 4(c).

\section{c. Photon autocorrelation analysis}

The data underling the photon autocorrelation measurement for the case of resonant excitation, displayed in Fig. 4(b), can be seen in Fig. 9(c). We compute the time differences between PSB clicks after splitting the signal and measuring with two photodetectors, and integrate the counts for the time differences for a narrow time window that avoids detector after-pulsing effects. We then fit a simple bunching model to the resulting data, as the green laser leads to a finite state initialization into a bright state (as is the case for off-resonant excitation measurements). Because of a finite number of 500 red pulses per measurement pulse sequence (see above), the normalization line in the data is not straight but sloped. By calculating the normalized coincidences at zero pulse separation (normalized to the point of the sloped line at zero pulse separation), we can then extract that the autocorrelation falls to $0.19 \pm 0.09$, or $0.16 \pm 0.07$ when correcting for background counts. This is well below the threshold of 0.5 , signaling that we are looking at emission from a single quantum emitter. All three resonant excitation photon autocorrelation measurements of $\mathrm{N}-V$ centers in this study fell well below 0.5 , thus confirming that the emission peaks in photoluminescence excitation scans are originating from single quantum emitters.

\section{APPENDIX E: CAVITY VIBRATIONS AND THEIR INFLUENCE ON COUNTS AND LIFETIMES}

All measurements in this paper are taken in a closedcycle cryostat operated at $T \sim 4 \mathrm{~K}$. Such a cryogenic system has intrinsic vibrations due to moving parts. Nevertheless, we choose a closed-cycle system - as opposed to an essentially vibration-free liquid helium bath cryostat - because of its ease of operation, and the possibility of uninterrupted measurement cycles on a timescale of several months without any human intervention; this is an important operational consideration for a future quantum network, with nodes distributed over distant locations, as it removes the cost and labor associated with a helium infrastructure.

\section{Characterization of root-mean-squared cavity vibrations}

To characterize the cavity length fluctuations induced by the cryostat, we follow the measurement principle schematically depicted in Fig. 10(a). We operate the red excitation laser on the flank of the cavity transmission, which allows us to map vibration-induced shifts in cavity frequency to cavity transmission changes. We lower the cavity finesse by retracting the fiber, which increases the beam diameter at the position of the fiber mirror to a value where the light mode in the cavity clips at the finite diameter of the ablated mirror region, introducing additional cavity losses. This broadens the Lorentzian cavity transmission to a width where the change in cavity length induced by vibrations in the cryostat is fully contained within one flank of the cavity transmission peak [indicated in red in Fig. 10(a)]. By measuring the Lorentzian cavity transmission function independently, we can then map changes in cavity transmission to changes in cavity detuning and thus cavity length.

The rms vibrations for a measurement time of $10 \mathrm{~s}$, binned into $50 \mathrm{~ms}$ time bins, are displayed in Fig. 10(b). We observe a (5-6)-fold change in vibration value over the course of the measurement. Utilizing a synchronization signal from the cryostat coldhead signaling the beginning of a new coldhead movement period, we can average the data with respect to time in the cryostat cycle; see Fig. 10(c). The square root of the integrated noise power for the full dataset is plotted in Fig. 10(d). There are several frequencies up to about $26 \mathrm{kHz}$ that contribute significantly to the observed vibrations. The multitude of peaks above the resonance frequency of our cavity piezos (about $2-4 \mathrm{kHz}$ ) make active cavity stabilization hard in practice. We therefore operate the cavity free running during all measurements, only correcting for slow drift by stabilizing the cavity to the excitation laser about every $10-20 \mathrm{~s}$. 

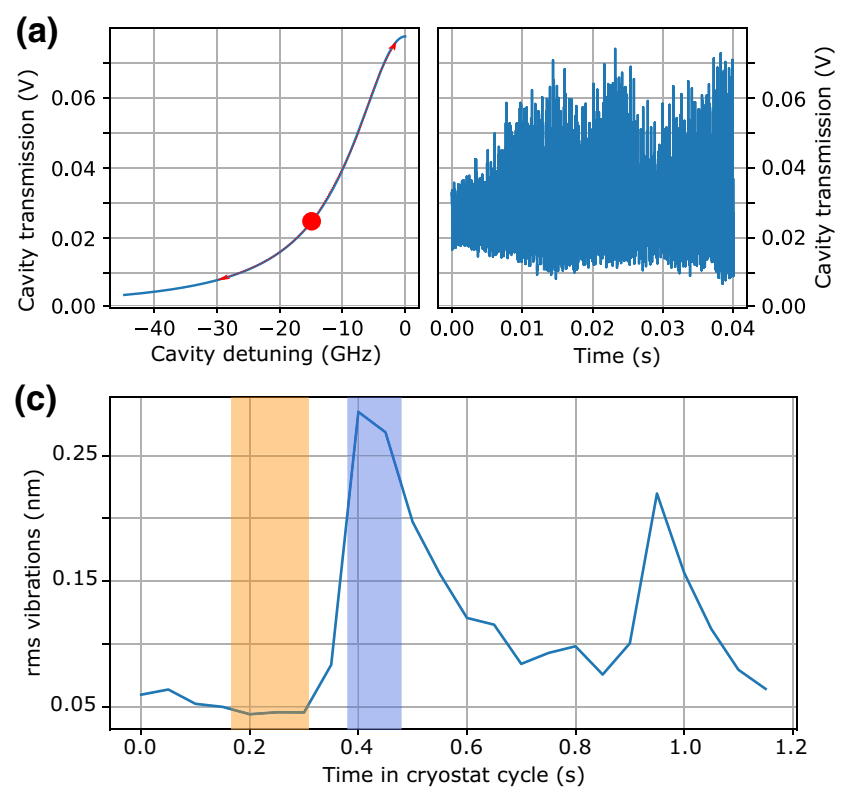

(b)

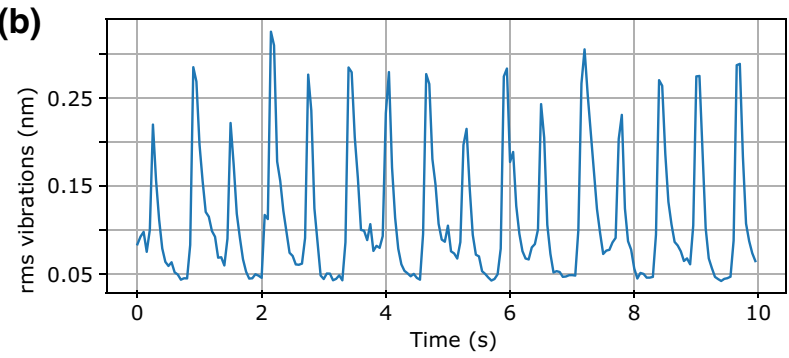

(d)

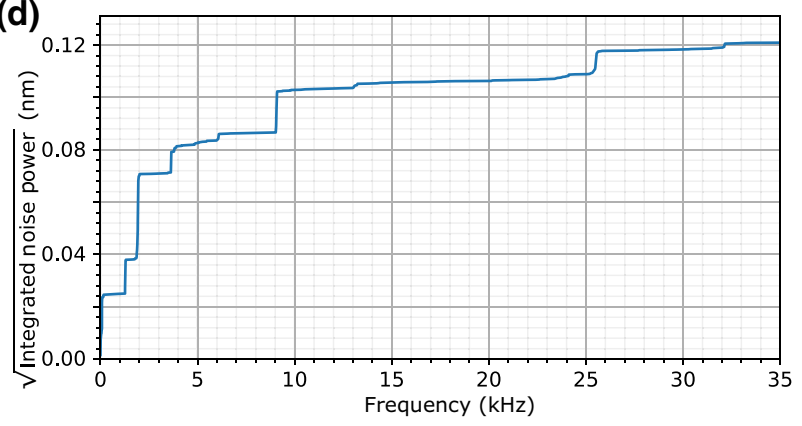

FIG. 10. Characterization of vibrations in the closed-cycle cryostat at $4 \mathrm{~K}$. (a) Measurement principle. The laser is operated on the flank of the cavity transmission (indicated with a red dot), such that vibration-induced shifts in cavity frequency cause a change in cavity transmission. (b) Root-mean-squared (rms) vibrations over a $10 \mathrm{~s}$ measurement cycle, binned into $50 \mathrm{~ms}$ time bins. (c) Using a synchronization signal of the cryostat marking the beginning of a new coldhead cycle, the same data as in (b) are averaged with respect to time in the cryostat cycle. Blue and orange shaded regions indicate time periods assigned as having low and high vibration values for data analysis similar to that in Fig. 11. (d) Square root of integrated noise power versus cavity frequency, showing the frequency contents of the observed vibrations.

\section{Influence on fluorescence counts and lifetimes}

As discussed in Appendix C, cavity length fluctuations influence the collected counts and detected lifetime from a $\mathrm{N}-V$ center. In the case of resonant excitation and PSB detection, both the excitation probability, and the Purcell enhancement, depend on the cavity detuning at a given time. To probe this dependence, we utilize a synchronization signal from the cryostat coldhead that marks the beginning of a new period, and use it to record the time in the cryostat period that a $\mathrm{N}-V$ center photon is detected. We assign $\mathrm{N}-V$ center photon arrival timestamps to periods in the cryostat with low [orange shaded area in Fig. 10(c)] and high vibration values [blue shaded area in Fig. 10(c)]. We can then extract the vibration influence on counts and corresponding excited state lifetime; see Fig. 11. During the cryostat times with low vibrations, we extract a N- $V$ center lifetime (counts per excitation pulse) of $9.77 \pm 0.08 \mathrm{~ns}$ $\left[(5.8 \pm 0.2) \times 10^{-4}\right]$, compared to $10.02 \pm 0.12 \mathrm{~ns}[(2.5 \pm$ $0.1) \times 10^{-4}$ ] during high vibration times. In comparison, the lifetime (counts) over all cryostat times is $9.87 \pm 0.04$ ns $\left[(4.7 \pm 0.2) \times 10^{-4}\right]$. This serves as further proof that it is indeed Purcell enhancement through coupling to a cavity that leads to a lifetime reduction and increase in counts of the $\mathrm{N}-V$ center when the cavity frequency is tuned to the $\mathrm{N}$ $V$ center transition frequency. Additionally, it also shows that reducing the vibrations in a future system increases
Purcell enhancement, as discussed in Appendix F. We average over all cryostat period times in the measurements in this study, except if stated otherwise.

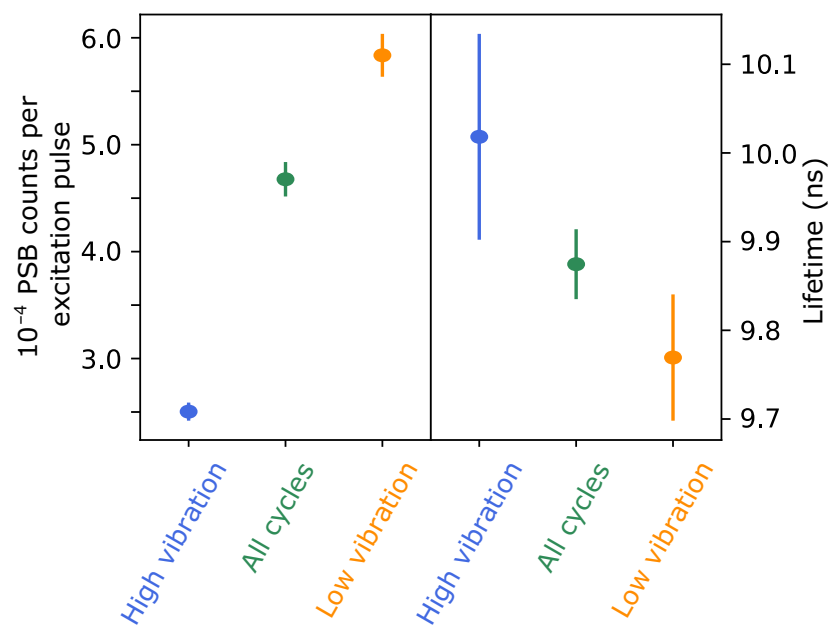

FIG. 11. Influence of the cavity vibrations on PSB counts per excitation pulse (left-hand side) and lifetime (right-hand side) for the case of resonant $\mathrm{N}-V$ center excitation. The cavity is kept on resonance with the $\mathrm{N}-V$ center transition frequency. The data are taken for a different cooldown and thus vibration level assignment than that depicted schematically in Fig. 10(c). 


\section{APPENDIX F: CURRENT CHALLENGES AND FUTURE IMPROVEMENTS}

In Sec. VI, we measured the absolute counts from a N- $V$ center in the cavity in both the PSB and the ZPL under resonant excitation. It is helpful to understand the loss contributions that prevent perfect PSB and ZPL detection. In this way, we can project what improvements may be possible with future upgrades to the setup. The investigations in this section underlie the loss plot in Fig. 5(b).

\section{Vibrations}

First, we estimate the effect of vibrations on cavity coupling and enhancement. In our model in Appendix C, the vibrations reduce three parameters: the excitation probability of the $\mathrm{N}-V$ center, the ZPL fraction emitted into the cavity, and the collection efficiency of the cavity. These effects are correlated, because the vibrations are slow (about millisecond timescale) compared to the excitation and emission timescales of the $\mathrm{N}-V$ center (about nanosecond timescale). Only the excitation probability of the N- $V$ center has a notable contribution to the PSB counts, as the fraction into the PSB is only slightly decreased for our cavity, and the PSB is not collected resonantly with the cavity.

From the data in Figs. 4(b) and 5(a), we determine that $(7.0 \pm 3.4) \%$ of the emission goes into the ZPL path, and we excite and detect ZPL photons with a probability of $(9.3 \pm 0.2) \times 10^{-5}$. If we set the vibration level to zero in our model, we find that $17 \%$ is emitted into the ZPL, and $1.7 \times 10^{-3} \mathrm{ZPL}$ photons are collected. Thus, the total reduction of ZPL collection and excitation due to vibrations is $13 \mathrm{~dB}$. Because these reductions are correlated, we distribute the vibration contributions to the losses in Fig. 5(b) according to the relative contribution to the $P^{5 / 2}$ term in Eq. (C14).

\section{Collection efficiency}

We divide the ZPL collection efficiency into two parts: internal collection efficiency and external collection efficiency $\left(\eta_{\mathrm{ZPL}}=\eta_{\text {int }} \eta_{\text {ext }}\right)$. The external collection efficiency is the coupling between the mode exiting the cavity and the detector, and is determined by the classical optics in between. The internal collection efficiency is the probability that a photon escapes the cavity and couples into the free-space mode.

The internal efficiency, $\eta_{\text {int }}$, can be estimated using the measured transmission rate of the free-space mirror, $\kappa_{\mathrm{FS}}$, as $\eta_{\text {int }}=\kappa_{\mathrm{FS}} / \kappa$. We design $\kappa_{\mathrm{FS}}$ to be significantly larger than the fiber transmission and scattering loss rates. However, the angle of the fiber forced us to operate in a regime where diffraction losses contributed significantly to the cavity finesse (which can be readily overcome with a new fiber). Therefore, in this work we operated with $\eta_{\text {int }}$ in the range of $0.05-0.17$, which also includes a factor of about $\frac{1}{3}$ from vibrations.

To measure the external collection efficiency, we send classical light through the fiber, and estimate the losses at each section between the fiber input and the detector. The loss to and from the cavity, probed in the fiber in reflection, is approximately $-12.4 \mathrm{~dB}$, including the fiber connector and fiber splice twice, and the reflection off the cavity once. Based on the depth of the reflection dip from the cavity, we estimate that we have a $2.8 \%$ incoupling efficiency. Based on the design values of our mirrors, we expect $2.5 \%$ incoupling from the fiber side, which is in reasonable agreement, and suggests that there is not a large mode mismatch between the cavity and the fiber. For a measurement of the transmitted light, we filter the input laser beam with a $20 \mathrm{~dB}$ filter and measure counts on an APD in the ZPL path. Subtracting out the light lost in the excitation path, we estimate that the total collection efficiency is $4 \%$. This is likely divided into approximately $11 \%$ internal collection efficiency and approximately $32 \%$ external collection efficiency, but the precise contributions are not known. The external efficiency is determined by classical optics and the detector efficiency, so we expect that the external efficiency can also be improved in future experiments with better coatings and superconducting nanowire detectors.

We can also estimate the collection efficiency of the PSB path to make full use of the direct comparison in counts between PSB and ZPL. This efficiency is relatively low in our setup, because we have a long working distance objective with a numerical aperture of 0.55 (Appendix A contains a full description of the optics path). Depending on the angle of the $\mathrm{N}-V$ center dipole emission axis in our $\langle 100\rangle$ sample, the collection is between $0.9 \%$ and $2.5 \%$. There is likely a bias towards $\mathrm{N}-V$ centers with a better coupled dipole, because they produce more collectable PSB counts, and thus are easier to spot when searching for $\mathrm{N}-V$ centers. The top collecting mirror has a narrow stop band, which by design allows $83 \%$ of the PSB light through. We estimate $83 \%$ transmission through the path and $70 \%$ APD detection efficiency, but the exact values are unknown. Altogether we expect between $0.4 \%$ and $1.2 \%$ collection efficiency in the PSB.

\section{Benchmarking with excitation probability correction}

We can benchmark our system against other diamond collection optics such as solid immersion lenses. However, the state of the art in these systems is near perfect initialization and excitation of the $\mathrm{N}-V$ center. Higher initialization can be achieved with an additional spin pumping laser and microwaves [21]. In our system we initialize with a green laser, which only has a limited probability of initializing into the $m_{s}=0$ spin state. Furthermore, we do not saturate the initialization with green or the excitation with 
red, due to limited laser power, so it is hard to estimate the probability of excitation. One might expect a lower laser power requirement than for noncavity $\mathrm{N}-V$ systems, because the $\mathrm{N}-V$ driving is enhanced by a factor of the finesse (about 2000). However, this is offset by losses in the fiber (about 0.24) and the low coupling efficiency into the cavity through the fiber mirror (about 0.025 ). The extended mode area in the fiber cavity compared to the mode of a high numerical aperture objective also reduces the driving (about 0.01). In total, we require approximately a factor of 10 more power, which is consistent with the probability of excitation we observe. Therefore, the counts we observe are not directly comparable to state of the art.

We can instead use our calculations of the collection efficiencies to estimate the excitation probability based on the total counts we observe from the $\mathrm{N}-V$ center in the PSB and the ZPL. First we use the ZPL counts; we expect that with perfect initialization the counts in the ZPL should be $F_{P}^{\mathrm{ZPL}} \beta_{0} /\left(F_{P}^{\mathrm{ZPL}} \beta_{0}+1\right) \eta_{\text {int }} \eta_{\text {ext }}$ or approximately $2.6 \times 10^{-3}$ based on the results of Secs. V and VI. This corresponds to an excitation probability of $3 \%$. For comparison, the PSB counts should be $\left(1-\beta_{0}\right) /\left(F_{P}^{\mathrm{ZPL}} \beta_{0}+1\right) \eta_{\mathrm{PSB}}$. The estimated collection efficiency and measured counts give us an excitation probability between and $14 \%$ and $4 \%$ (with a bias towards the latter). This corresponds to between 0.6 and $2 \times 10^{-3}$ counts in the ZPL with perfect initialization. The estimates show reasonable agreement, and exact determination of the different losses is left for future work. Even with the limited collection efficiency in this work, the ZPL counts we collect are comparable to state of the art when correcting for initialization and excitation probability.

\section{Improvements}

Based on the current challenges and our modeling, we suggest three improvements that should be possible in the short term, as summarized in Table II. We constrain ourselves to performances that have already been achieved in other systems. The first improvement is to implement microwave control of the ground state $\mathrm{N}-V$ center spin, which will allow spin pumping and also yellow resonant (a)

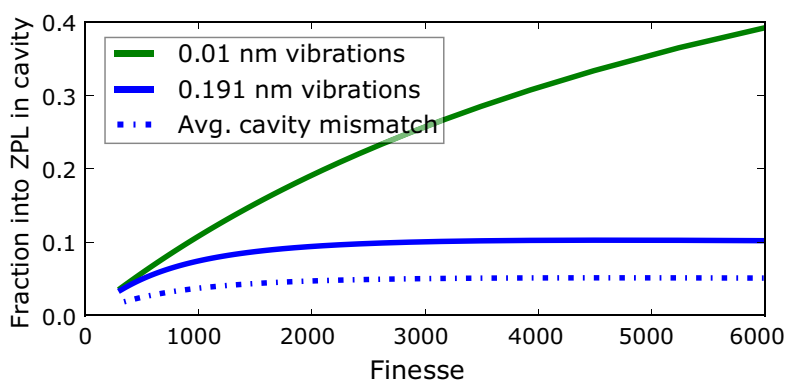

(b)

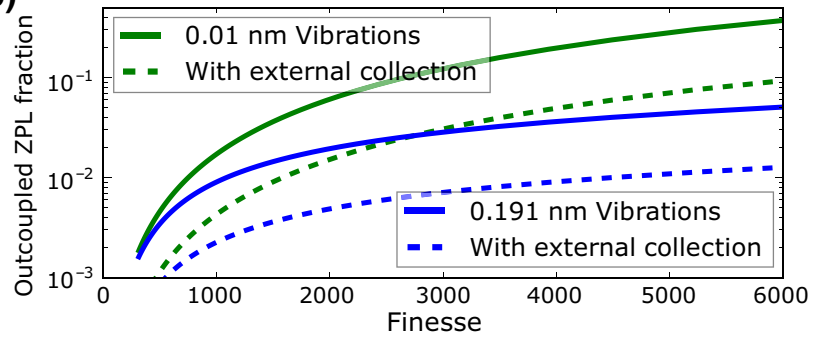

FIG. 12. Projections for ZPL emission and collection fraction. We fix the design parameters of the mirrors to match our current system (Finesse 6200). Fraction emitted into the ZPL (a) and outcoupled fraction of photons in the ZPL (b) for the current (blue) and improved (green) vibration levels, as a function of achieved cavity finesse. These simulations are based on the model from Ref. [45].

repumping for higher initialization and even lower spectral diffusion. Using these techniques, it should be possible to achieve near unity excitation, as has been shown in bulk diamond [21,47].

The second improvement is to reduce vibrations; a number of research groups have achieved considerably lower vibrations of comparable fiber-based microcavities [14,51-53]. In Fig. 12, we simulate the dependence of ZPL emission fraction and collection fraction on the finesse for different vibration levels. We project that a factor of 20 vibration levels reduction gains a factor of 16 in total ZPL collection.

TABLE II. Summary of suggested improvements to the current diamond fiber cavity. The first three upgrades represent improvements that have already been achieved in other systems, as indicated in the second column. Finesse would be limited by the mirror if achieved losses limit the finesse to significantly greater than 6000. The last two items have been achieved, but not simultaneously with the other requirements. We give the resulting enhancement of the ZPL collected, the absolute fraction collected in the ZPL, the cooperativity, and the relative decrease in the excitation power required.

\begin{tabular}{|c|c|c|c|c|c|}
\hline Improvement & $\begin{array}{l}\text { Achieved } \\
\text { in Refs. }\end{array}$ & $\begin{array}{c}\text { Enhancement } \\
\text { factor }\end{array}$ & $\begin{array}{c}\text { ZPL\% } \\
\text { collection }\end{array}$ & $\begin{array}{c}\text { Cooperativity } \\
C\end{array}$ & $\begin{array}{l}\text { Excitation } \\
\text { power }\end{array}$ \\
\hline Spin pump and resonant repump & {$[21,47]$} & 20 & 0.2 & 0.08 & 1 \\
\hline $20 \times$ Vibration reduction & {$[14,51-53]$} & 16 & 3 & 0.21 & 0.55 \\
\hline Finesse limited by mirror coatings (6000) & {$[39,42]$} & 3 & 9 & 0.64 & 0.18 \\
\hline Higher finesse (11 000) & {$[39,42]$} & 1.2 & 10 & 1.0 & 0.1 \\
\hline Diamondlike mode & {$[39,42,43]$} & 2 & 20 & 1.5 & 0.06 \\
\hline
\end{tabular}


The third improvement is increasing the finesse; our current finesse is limited by diffraction losses, but with a better fiber it should be limited by the mirror coatings, gaining another factor of 3. As indicated in Table II, the laser power required to saturate the excitation of $\mathrm{N}-V$ centers in the cavity should reduce by a factor of 5 after these improvements. Furthermore, the ZPL emission fraction is expected to increase by a factor 5 , reducing the required polarization extinction by a factor of 25 . Although it has not yet been achieved simultaneously, a diamond fiber cavity with finesse 11000 diamondlike modes would further increase ZPL collection by approximately a factor of 2. Altogether, we expect that about $10 \%$ ZPL collection should be possible with already achieved parameters and $20 \%$ with combining the best results from different setups. The improvements in the Purcell factor also correspond to an increase in the cooperativity $C$, and there is a route to exceeding $C=1$ with this approach.

[1] H. J. Kimble, The quantum internet, Nature 453, 1023 (2008).

[2] S. Wehner, D. Elkouss, and R. Hanson, Quantum internet: A vision for the road ahead, Science 362, 303 (2018).

[3] A. Ekert and R. Renner, The ultimate physical limits of privacy, Nature 507, 443 (2014).

[4] N. H. Nickerson, J. F. Fitzsimons, and S. C. Benjamin, Freely Scalable Quantum Technologies Using Cells of 5To-50 Qubits with Very Lossy and Noisy Photonic Links, Phys. Rev. X 4, 1 (2014).

[5] D. Gottesman, T. Jennewein, and S. Croke, LongerBaseline Telescopes Using Quantum Repeaters, Phys. Rev. Lett. 109, 1 (2012).

[6] C. T. Nguyen, D. D. Sukachev, M. K. Bhaskar, B. Machielse, D. S. Levonian, E. N. Knall, P. Stroganov, R. Riedinger, H. Park, M. Lončar, and M. D. Lukin, Quantum Network Nodes Based on Diamond Qubits with an Efficient Nanophotonic Interface, Phys. Rev. Lett. 123, 1 (2019).

[7] M. K. Bhaskar, R. Riedinger, B. Machielse, D. S. Levonian, C. T. Nguyen, E. N. Knall, H. Park, D. Englund, M. Lončar, D. D. Sukachev, and M. D. Lukin, Experimental demonstration of memory-enhanced quantum communication, Nature 580, 60 (2020).

[8] M. E. Trusheim et al., Transform-Limited Photons from a Coherent Tin-Vacancy Spin in Diamond, Phys. Rev. Lett. 124, 1 (2020).

[9] D. J. Christle, P. V. Klimov, C. F. de las Casas, K. Szász, V. Ivády, V. Jokubavicius, J. U. Hassan, M. Syväjärvi, W. F. Koehl, T. Ohshima, N. T. Son, E. Janzén, Á. Gali, and D. D. Awschalom, Isolated Spin Qubits in SiC with a HighFidelity Infrared Spin-To-Photon Interface, Phys. Rev. X 7, 021046 (2017).

[10] R. Nagy, M. Niethammer, M. Widmann, Y.-C. Chen, P. Udvarhelyi, C. Bonato, J. U. Hassan, R. Karhu, I. G. Ivanov, N. T. Son, J. R. Maze, T. Ohshima, Ö. O. Soykal, Á. Gali, S.-Y. Lee, F. Kaiser, and J. Wrachtrup, High-fidelity spin and optical control of single silicon-vacancy centres in silicon carbide, Nat. Commun. 10, 1954 (2019).
[11] A. Bourassa, C. P. Anderson, K. C. Miao, M. Onizhuk, H. Ma, A. L. Crook, H. Abe, J. Ul-Hassan, T. Ohshima, N. T. Son, G. Galli, and D. D. Awschalom, Entanglement and control of single nuclear spins in isotopically engineered silicon carbide, Nat. Mater. 19, 1319 (2020).

[12] M. Raha, S. Chen, C. M. Phenicie, S. Ourari, A. M. Dibos, and J. D. Thompson, Optical quantum nondemolition measurement of a single rare earth ion qubit, Nat. Commun. 11, 1 (2020).

[13] J. M. Kindem, A. Ruskuc, J. G. Bartholomew, J. Rochman, Y. Q. Huan, and A. Faraon, Control and single-shot readout of an ion embedded in a nanophotonic cavity, Nature 580, 201 (2020).

[14] B. Merkel, A. R Ulanowski, and A. Reiserer, Coherent and Purcell-Enhanced Emission from Erbium Dopants in a Cryogenic High-Q Resonator, Phys. Rev. X 10, 041025 (2020).

[15] A. Delteil, Z. Sun, W. B. Gao, E. Togan, S. Faelt, and A. Imamoglu, Generation of heralded entanglement between distant hole spins, Nat. Phys. 12, 218 (2016).

[16] R. Stockill, M. J. Stanley, L. Huthmacher, E. Clarke, M. Hugues, A. J. Miller, C. Matthiesen, C. Le Gall, and M. Atatüre, Phase-Tuned Entangled State Generation between Distant Spin Qubits, Phys. Rev. Lett. 119, 1 (2017).

[17] S. Ritter, C. Nölleke, C. Hahn, A. Reiserer, A. Neuzner, M. Uphoff, M. Mücke, E. Figueroa, J. Bochmann, and G. Rempe, An elementary quantum network of single atoms in optical cavities, Nature 484, 195 (2012).

[18] J. Hofmann, M. Krug, N. Ortegel, L. Gérard, M. Weber, W. Rosenfeld, and H. Weinfurter, Heralded entanglement between widely separated atoms, Science 337, 72 (2012).

[19] D. Hucul, I. V. Inlek, G. Vittorini, C. Crocker, S. Debnath, S. M. Clark, and C. Monroe, Modular entanglement of atomic qubits using photons and phonons, Nat. Phys. 11, 37 (2015).

[20] L. J. Stephenson, D. P. Nadlinger, B. C. Nichol, S. An, P. Drmota, T. G. Ballance, K. Thirumalai, J. F. Goodwin, D. M. Lucas, and C. J. Ballance, High-Rate, High-Fidelity Entanglement of Qubits Across an Elementary Quantum Network, Phys. Rev. Lett. 124, 1 (2020).

[21] H. Bernien, B. Hensen, W. Pfaff, G. Koolstra, M. S. Blok, L. Robledo, T. H. Taminiau, M. Markham, D. J. Twitchen, L. Childress, and R. Hanson, Heralded entanglement between solid-state qubits separated by three metres, Nature 497, 86 (2013).

[22] C. E. Bradley, J. Randall, M. H. Abobeih, R. C. Berrevoets, M. J. Degen, M. A. Bakker, M. Markham, D. J. Twitchen, and T. H. Taminiau, A Ten-Qubit Solid-State Spin Register with Quantum Memory up to One Minute, Phys. Rev. X 9, 31045 (2019).

[23] N. Kalb, A. A. Reiserer, P. C. Humphreys, J. J. W. Bakermans, S. J. Kamerling, N. H. Nickerson, S. C. Benjamin, D. J. Twitchen, M. Markham, and R. Hanson, Entanglement distillation between solid-state quantum network nodes, Science 356, 928 (2017).

[24] P. C. Humphreys, N. Kalb, J. P. J. Morits, R. N. Schouten, R. F. L. Vermeulen, D. J. Twitchen, M. Markham, and R. Hanson, Deterministic delivery of remote entanglement on a quantum network, Nature 558, 268 (2018). 
[25] B. Hensen, H. Bernien, A. E. Dréau, A. Reiserer, N. Kalb, M. S. Blok, J. Ruitenberg, R. F. L. Vermeulen, R. N. Schouten, C. Abellán, W. Amaya, V. Pruneri, M. W. Mitchell, M. Markham, D. J. Twitchen, D. Elkouss, S. Wehner, T. H. Taminiau, and R. Hanson, Loophole-free Bell inequality violation using electron spins separated by 1.3 kilometres, Nature 526, 682 (2015).

[26] A. Faraon, C. Santori, Z. Huang, V. M. Acosta, and R. G. Beausoleil, Coupling of Nitrogen-Vacancy Centers to Photonic Crystal Cavities in Monocrystalline Diamond, Phys. Rev. Lett. 109, 2 (2012).

[27] B. J. M. Hausmann, B. J. Shields, Q. Quan, Y. Chu, N. P. De Leon, R. Evans, M. J. Burek, A. S. Zibrov, M. Markham, D. J. Twitchen, H. Park, M. D. Lukin, and M. Loncar, Coupling of NV centers to photonic crystal nanobeams in diamond, Nano Lett. 13, 5791 (2013).

[28] L. Li, T. Schröder, E. H. Chen, M. Walsh, I. Bayn, J. Goldstein, O. Gaathon, M. E. Trusheim, M. Lu, J. Mower, M. Cotlet, M. L. Markham, D. J. Twitchen, and D. Englund, Coherent spin control of a nanocavity-enhanced qubit in diamond, Nat. Commun. 6, 6173 (2015).

[29] J. Riedrich-Möller, S. Pezzagna, J. Meijer, C. Pauly, F. Mücklich, M. Markham, A. M. Edmonds, and C. Becher, Nanoimplantation and Purcell enhancement of single nitrogen-vacancy centers in photonic crystal cavities in diamond, Appl. Phys. Lett. 106, 2 (2015).

[30] T. Schröder, M. Walsh, J. Zheng, S. Mouradian, L. Li, G. Malladi, H. Bakhru, M. Lu, A. Stein, M. Heuck, and D. Englund, Scalable fabrication of coupled NV center - photonic crystal cavity systems by self-aligned $\mathrm{N}$ ion implantation, Opt. Mater. Express 7, 1514 (2017).

[31] T. Jung, J. Görlitz, B. Kambs, C. Pauly, N. Raatz, R. Nelz, E. Neu, A. M. Edmonds, M. Markham, F. Mücklich, J. Meijer, and C. Becher, Spin measurements of NV centers coupled to a photonic crystal cavity, APL Photonics 4, 120803 (2019).

[32] A. Faraon, P. E. Barclay, C. Santori, K. M. C. Fu, and R. G. Beausoleil, Resonant enhancement of the zero-phonon emission from a colour centre in a diamond cavity, Nat. Photonics 5, 301 (2011).

[33] D. Riedel, I. Söllner, B. J. Shields, S. Starosielec, P. Appel, E. Neu, P. Maletinsky, and R. J. Warburton, Deterministic Enhancement of Coherent Photon Generation from a Nitrogen-Vacancy Center in Ultrapure Diamond, Phys. Rev. X 7, 1 (2017).

[34] S. Johnson, P. R. Dolan, T. Grange, A. A. P. Trichet, G. Hornecker, Y. C. Chen, L. Weng, G. M. Hughes, A. A. R. Watt, A. Auffèves, and J. M. Smith, Tunable cavity coupling of the zero phonon line of a nitrogen-vacancy defect in diamond, New J. Phys. 17, 122003 (2015).

[35] S. B. Van Dam, M. Walsh, M. J. Degen, E. Bersin, S. L. Mouradian, A. Galiullin, M. Ruf, M. IJspeert, T. H. Taminiau, R. Hanson, and D. R. Englund, Optical coherence of diamond nitrogen-vacancy centers formed by ion implantation and annealing, Phys. Rev. B 99, 161203 (2019).

[36] M. Kasperczyk, J. A. Zuber, A. Barfuss, J. Kölbl, V. Yurgens, S. Flågan, T. Jakubczyk, B. Shields, R. J. Warburton, and P. Maletinsky, Statistically modeling optical linewidths of nitrogen vacancy centers in microstructures, Phys. Rev. B 102, 075312 (2020).
[37] M. Ruf, M. IJspeert, S. Van Dam, Nick De Jong, H. Van Den Berg, Guus Evers, and Ronald Hanson, Optically coherent nitrogen-vacancy centers in micrometerthin etched diamond membranes, Nano Lett. 19, 3987 (2019).

[38] E. Janitz, M. Ruf, M. Dimock, A. Bourassa, J. Sankey, and L. Childress, Fabry-Perot microcavity for diamond-based photonics, Phys. Rev. A 92, 1 (2015).

[39] S. Bogdanović, S. B. van Dam, C. Bonato, L. C. Coenen, A.-M. J. Zwerver, B. Hensen, M. S. Z. Liddy, T. Fink, A. Reiserer, M. Lončar, and R. Hanson, Design and lowtemperature characterization of a tunable microcavity for diamond-based quantum networks, Appl. Phys. Lett. 110, 171103 (2017)

[40] S. Bogdanović, Madelaine S. Z. Liddy, S. B. van Dam, Lisanne C. Coenen, T. Fink, Marko Lončar, and Ronald Hanson, Robust nano-fabrication of an integrated platform for spin control in a tunable microcavity, APL Photonics 2 , 126101 (2017).

[41] S. Häußler, J. Benedikter, K. Bray, B. Regan, A. Dietrich, J. Twamley, I. Aharonovich, D. Hunger, and A. Kubanek, Diamond photonics platform based on silicon vacancy centers in a single-crystal diamond membrane and a fiber cavity, Phys. Rev. B 99, 1 (2019).

[42] R. Høy Jensen, E. Janitz, Y. Fontana, Y. He, O. Gobron, I. P. Radko, M. Bhaskar, R. Evans, C. D. R. Rosenblueth, L. Childress, A. Huck, and U. L. Andersen, CavityEnhanced Photon Emission from a Single GermaniumVacancy Center in a Diamond Membrane, Phys. Rev. Appl. 13, 064016 (2020).

[43] M. Salz, Y. Herrmann, A. Nadarajah, A. Stahl, M. Hettrich, A. Stacey, S. Prawer, D. Hunger, and F. Schmidt-Kaler, Cryogenic platform for coupling color centers in diamond membranes to a fiber-based microcavity, Appl. Phys. B 126, 131 (2020).

[44] D. Hunger, T. Steinmetz, Y. Colombe, C. Deutsch, T. W. Hänsch, and J. Reichel, A fiber Fabry-Perot cavity with high finesse, New J. Phys. 12, 065038 (2010).

[45] S. B. van Dam, M. Ruf, and R. Hanson, Optimal design of diamond-air microcavities for quantum networks using an analytical approach, New J. Phys. 20, 115004 (2018).

[46] D. Riedel, S. Flågan, P. Maletinsky, and R. J. Warburton, Cavity-Enhanced Raman Scattering for in Situ Alignment and Characterization of Solid-State Microcavities, Phys. Rev. Appl. 13, 1 (2020).

[47] L. Robledo, L. Childress, H. Bernien, B. Hensen, P. F. A. Alkemade, and R. Hanson, High-fidelity projective read-out of a solid-state spin quantum register, Nature 477, 574 (2011).

[48] N. Kalb, P. C. Humphreys, J. J. Slim, and R. Hanson, Dephasing mechanisms of diamond-based nuclearspin memories for quantum networks, Phys. Rev. A 97, 1 (2018).

[49] H. Bernien, L. Childress, L. Robledo, M. Markham, D. Twitchen, and R. Hanson, Two-Photon Quantum Interference from Separate Nitrogen Vacancy Centers in Diamond, Phys. Rev. Lett. 108, 043604 (2012).

[50] L. Robledo, H. Bernien, I. Van Weperen, and R. Hanson, Control and Coherence of the Optical Transition of Single Nitrogen Vacancy Centers in Diamond, Phys. Rev. Lett. 105, 1 (2010). 
[51] B. Casabone, C. Deshmukh, S. Liu, D. Serrano, A. Ferrier, T. Hümmer, P. Goldner, D. Hunger, and H. de Riedmatten, Dynamic control of Purcell enhanced emission of erbium ions in nanoparticles, arXiv:2001.08532 (2020)

[52] L. Greuter, S. Starosielec, D. Najer, A. Ludwig, L. Duempelmann, D. Rohner, and R. J. Warburton, A small mode volume tunable microcavity: Development and characterization, Appl. Phys. Lett. 105, 121105 (2014).

[53] J. F. S. Brachmann, H. Kaupp, T. W. Hänsch, and D. Hunger, Photothermal effects in ultra-precisely stabilized tunable microcavities, Opt. Express 24, 21205 (2016).

[54] E. Janitz, M. Ruf, Y. Fontana, J. Sankey, and L. Childress, High mechanical bandwidth fiber-coupled Fabry-Perot cavity, Opt. Express 25, 20932 (2017).

[55] G. Murta, S. B. van Dam, J. Ribeiro, R. Hanson, and S. Wehner, Towards a realization of device-independent quantum key distribution, Quantum Sci. Technol. 4, 035011 (2019).

[56] F. Rozpedek, R. Yehia, K. Goodenough, M. Ruf, P. C. Humphreys, R. Hanson, S. Wehner, and D. Elkouss, Nearterm quantum-repeater experiments with nitrogen-vacancy centers: Overcoming the limitations of direct transmission, Phys. Rev. A 99, 052330 (2019).

[57] M. Ruf, M. J. Weaver, S. B. van Dam, and R. Hanson, Data for: "Resonant Excitation and Purcell Enhancement of Coherent Nitrogen-Vacancy Centers Coupled to a Fabry-
Perot Microcavity," https://doi.org/10.4121/13537112 (2021).

[58] A. Reiserer and G. Rempe, Cavity-based quantum networks with single atoms and optical photons, Rev. Mod. Phys. 87, 1379 (2015).

[59] M. W. Doherty, N. B. Manson, P. Delaney, F. Jelezko, J. Wrachtrup, and L. C. L. Hollenberg, The nitrogen-vacancy colour centre in diamond, Phys. Rep. 528, 1 (2013).

[60] J. Borregaard, A. S. Sørensen, and P. Lodahl, Quantum networks with deterministic spin-photon interfaces, Adv. Quantum Technol. 2, 1800091 (2019).

[61] M. L. Goldman, A. Sipahigil, M. W. Doherty, N. Y. Yao, S. D. Bennett, M. Markham, D. J. Twitchen, N. B. Manson, A. Kubanek, and M. D. Lukin, Phonon-Induced Population Dynamics and Intersystem Crossing in Nitrogen-Vacancy Centers, Phys. Rev. Lett. 114, 145502 (2015).

[62] C. Kurtsiefer, S. Mayer, P. Zarda, and H. Weinfurter, Stable Solid-State Source of Single Photons, Phys. Rev. Lett. 85, 290 (2000).

[63] G. Waldherr, J. Beck, M. Steiner, P. Neumann, A. Gali, T. H. Frauenheim, F. Jelezko, and J. Wrachtrup, Dark States of Single Nitrogen-Vacancy Centers in Diamond Unraveled by Single Shot NMR, Phys. Rev. Lett. 106, 1 (2011).

[64] N. Aslam, G. Waldherr, P. Neumann, F. Jelezko, and J. Wrachtrup, Photo-induced ionization dynamics of the nitrogen vacancy defect in diamond investigated by single-shot charge state detection, New J. Phys. 15, 013064 (2013). 